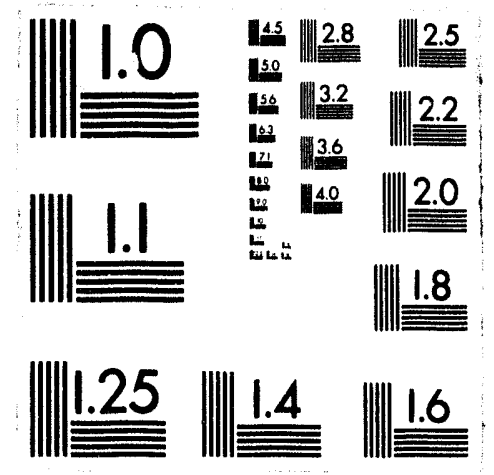



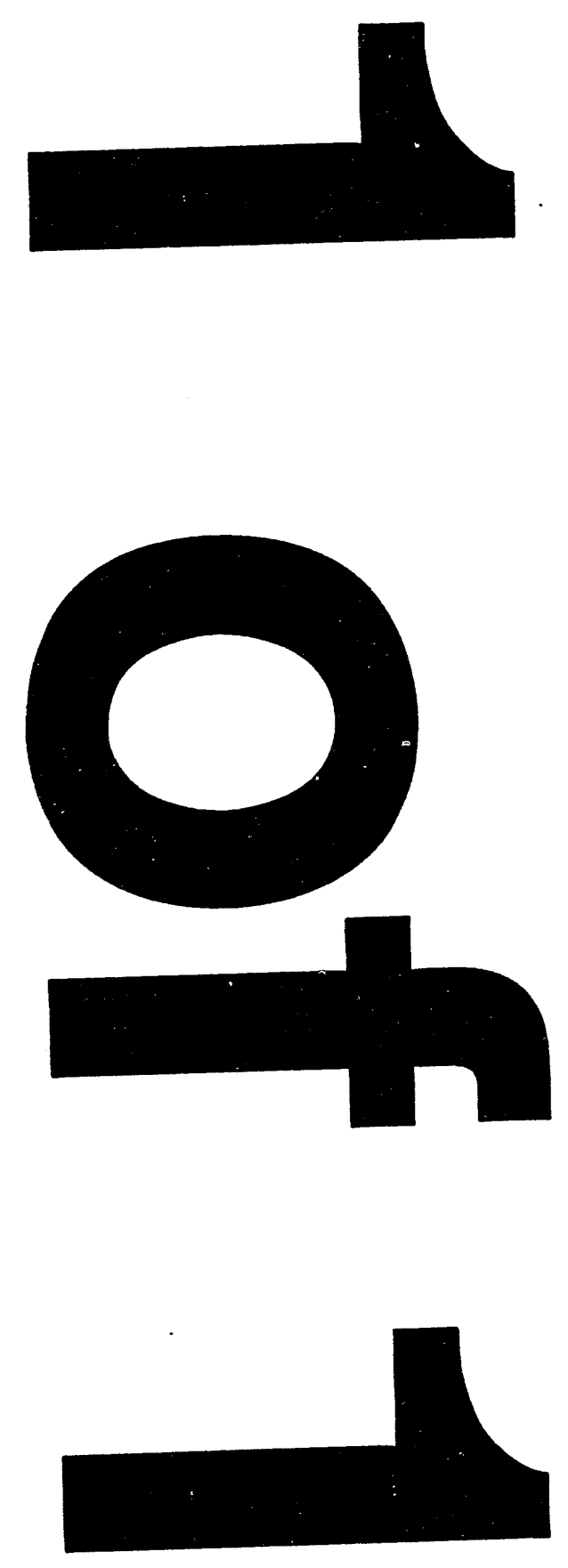


\section{Evaluation of High-Efficiency Gas-Liquid} Contactors for Natural Gas Processing

\section{Semi-Annual Report}

December 1993

Work Performed Under Contract No.: DE-FC21-92MC28178

\footnotetext{
For

U.S. Department of Energy

Office of Fossil Energy

Morgantown, West Virginia

By

Institute of Gas Technology

Chicago, Illinois
}

Morgantown Energy Technology Center 


\section{DISCLAIMER}

This report was prepared as an account of work sponsored by an agency of the United States Government. Neither the United States Government nor any agency thereof, nor any of their employees, makes any warranty, express or implied, or assumes any legal liability or responsibility for the accuracy, completeness, or usefulness of any information, apparatus, product, or process disclosed, or represents that its use would not infringe privately owned rights. Reference herein to any specific commercial product, process, or service by trade name, trademart, manufacturer, or otherwise does not necessarily constitute or imply its endorsement, recommendation, or favoring by the United States Government or any agency thereof. The views and opinions of authors expressed berein do not necessarily state or reflect those of the United States Government or any agency thereof.

This report has been reproduced directly from the best available copy.

Available to DOE and DOE contractors from the Office of Scientific and Technical Information, 175 Oak Ridge Tumpike, Oak Ridge, TN 37831; prices available at (615) 576-8401.

Available to the public from the National Tectnical Information Service, U.S. Department of Commerce, 5285 Port Royal Road, Springfield, VA 22161; phone orders accepted at (703) 487-4650. 


\section{Technical Progress Report (2nd Semi-Annual) \\ Cooperative Agreement No. DE-FC21-92MC28178 \\ Reporting Period: 4/01/93 - 09/30/93}

\section{PROJECT OBJECTIVE}

The objective of this proposed program is to evaluate the potential of rotating gas-liquid contactors for natural gas processing by expanding the currently available database. This expansion will focus on application of this technology to environments representative of those typically encountered in natural gas processing plants. Operational and reliability concerns will be addressed while generating pertinent engineering data relating to the mass-transfer process.

\section{WORK TO BE PERFORMED THIS REPORTING PERIOD}

- Complete all negotiations and processing of agreements.

- Complete assembly, modifications, shakedown, and conduct fluid dynamic studies using the plastic rotary contactor unit.

- Confirmation of project test matrix.

- Locate, and transport an amine plant and dehydration plant.

\section{WORK ACCOMPLISHED THIS REPORTING PERIOD}

\section{Task 1. National Environmental Policy Act}

This task was completed.

\section{Task 2. Field Experimental Site Selection}

Mr. L. Upshaw of Northern Illinois Gas Company was contacted as part of our effort to locate a potential field test site for the high pressure Higee TM Unit (China unit). Mr. Upshaw indicated that they might be interested in providing a site for dehydration testing, either a slip stream or a small well. Their aquifer natural gas storage facility produces 70 to 80 MMSCFD at an inlet pressure of 250 to 340 psia and temperature from $40^{\circ}$ to $50^{\circ} \mathrm{F}$. Individual wells range from 1 to $2 \mathrm{MMSCFD}$ flow rates. Northem Illinois Gas's involvement in the program depends on the results of IGT's studies, the fit of this technology to their operating conditions, the fit of this application to our research program, and ultimately the approval of the Technical Management Committee. 
IGT has also contacted Marathon and Texaco and will continue to search for other potential site hosts. The final decision will be made by the project Technical Management Committee which consists of DOE/METC, GRI, site host, Glitsch, and IGT. Glitsch and IGT are the non-voting members of this committee.

\section{Task 3. Field Experimental Skid Unit Desion And Preliminary Economic Evaluations}

This task was inactive.

\section{Task 4. Project Review}

This task was inactive.

\section{Task 5. Information Required for NEPA. Field Site}

This task was inactive.

\section{Task 6. Fluid Dynamic Studies}

Preparation of the facilities (electrical, heating, and plumbing) is continuing. During this period, assembly of the plastic rotary contactor unit was initiated. The unit is approximately $95 \%$ complete. IGT has requested and received approval to issue purchase orders for the variable frequency drive and blower required to complete the mechanical assembly of the plastic rotating contactor. When this equipment is received and the data acquisition system arrives from Glitsch, shake down and calibration of this unit will be conducted.

\section{Task 7. Mass-Transfer Coefficient Studies}

The mass-transfer coefficient experiments to be conducted under this program will be done using a high pressure Higee TM rotary contactor known as the "China Unit". Different sized "tors are used in this unit to change the flow capacities. General information on this unit is given in Table 1.

\section{Table 1. GENERAL INFORMATION ON GLITSCH'S "CHINA UNIT"}

$$
\begin{aligned}
& \text { Maximum Operating Pressure } \\
& \text { Potential Gas Compositions } \\
& \text { Large Rotor } \\
& \text { Maximum Gas Flows } \\
& \text { Minimum Gas Flows } \\
& \text { Smallest Rotor } \\
& \text { Maximum Gas Flows } \\
& \text { Minimum Gas Flows }
\end{aligned}
$$

1000 psig

Natural gas with sulphur compounds

1.0 MiMSCFD (41,667 SCFH)

0.25 MMSCFD (10,042 SCFH)

0.024 MMSCFD (1,000 SCFH)

360 SCFD (15 SCFH) 
Contractor Reports Receipt Coordinator Page 3
Technical Progress Report (2nd Semi-Annual)

Agreement No. DE-FC21-92MC28178

This unit has been operated at minimum gas flow for the smallest rotor, i.e. $15 \mathrm{SCFH}$, however operation at this level is not recommended. We anticipate designing our experiments to operate at or above $25 \%$ of minimum capacity for the rotor size used.

Dehydration Studies. It is anticipated that these experiments will be conducted using TEG and NFM solvents. Bids were requested for both a 0.5 and 1.0 MMSCFD glycol dehydration units from Glitsch Package Plants, Sivalls, Inc., B. S. \& B. Engineering Company, Inc, Petrofab, and Smith Industries. Bids received thus far for new plants range from $\$ 10,000$ to $\$ 35,000$ depending on solvent regeneration requirements. The higher the purity of solvent the greater the cost. These bids are being evaluated based suitability of the equipment to this program as well as cost.

Bulk Acid-Gas Removal. It is anticipated that a chemical and a physical solvent will be tested in the China Unit under this subtask. Table 2 shows a comparison of some of the properties of MEA, DEA, DGA, and MDEA. Gas processing simulations were conducted for MDEA, DEA, and MEA using Texaco Chemical Company's "QuickTreat TM: Gas Treating Preliminary Evaluation Program." This is not a rigorous equilibrium model for detailed design of a plant, however it does provide useful information for determining gas processing strategies. The subquality gas processing system IGT is developing will be designed to handle gas containing up to $15 \% \mathrm{CO}_{2}$ and $5 \% \mathrm{H}_{2} \mathrm{~S}$ for a maximum total of $20 \%$ acid-gas in the feed. This composition is higher then the typical range for use of chemical solvents but for research flexibility we want to cover the compositions of the majority of hie reserves in the U.S. containing acid gases. Figure 1 shows QuickTreat ${ }^{T M}$ 's estimation of solvent flow rate versus sour gas flow rate for a plant operating at 1,000 psia with the above gas composition. Figure 2 shows estimation of reboiler duty under the same conditions. From Table 2 and Figures 1 and 2 it is apparent why MDEA is becoming popular for gas processing. Compared to the other amines, MDEA has many favorable properties including better corrosion characteristics, lower solvent circulation rates and lower reboiler duty. We anticipate using MDEA as the chemical solvent and NFM as the physical solvent in the bulk acid gas removal studies with the China Unit. Appendix A contains inputs used for QuickTreat TM. Appendix B contains some a summary of calculations and Appendix $C$ has the output from the program.

Another request for information on availability of amine and dehydration plants was made to seven GRI advisors. Mr. Pat Knight of Texaco located a $10 \mathrm{gpm}$ amine plant. Mr. Knight is currently investigating the condition and availability of that plant. 
Contractor Reports Receipt Coordinator

Page 4
Technical Progress Report (2nd Semi-Annual)

Agreement No. DE-FC21-92MC28178

Table 2. TYPICAL OPERATING CONDITIONS AND DATA FOR AMINES

Amine

MEA

DEA

DGA

MDEA

Molecular Weight

61.08

105.14

105.14

119.16

Boiling Point, ${ }^{\circ} \mathbf{F}$

338.9

516.2

430

477

50.9

82.4

9.5

$-9.3$

Density, lb/gal @ 60F

8.48

9.09

8.82

8.68

Specific Heat @ 60% Btu/lb/\%F

0.608

0.600

0.571

0.535

Thermal Conductivity,

Btw/[(h-sq ft- $\left.\left.{ }^{\circ} \mathrm{F}\right) / \mathrm{ft}\right]$ @ $68^{\circ} \mathrm{F}$

0.148

0.127

0.121

0.159

Latent Heat of Vaporization, Btu/lb

355

288

219

204

Viscosity, cp

Solution Strength, wt \%

24.1@68F

15-20

$350 @ 68^{\circ} \mathrm{F}$

40 @ $60^{\circ} \mathrm{F}$

$1.3 @ 50^{\circ} \mathrm{F}$

pH Number

High

25-35

40-60

30-50

Acid Gas Loading, mole/mole

0.3-0.4

Medium

High

Medium

$\Delta \mathrm{H}_{\mathrm{r}}{ }^{*}$ for $\mathrm{H}_{2} \mathrm{~S}, \mathrm{Btu} / \mathrm{lb}$

550

0.3-0.4

0.3-0.4

Unlimited

$\Delta \mathrm{H}_{\mathrm{r}}^{*}$ for $\mathrm{CO}_{2}, \mathrm{Btu} / \mathrm{lb}$

825

Ability to Preferentially

Absorb $\mathrm{H}_{2} \mathrm{~S}$

Ability to Preferentially

Absorb $\mathrm{CO}_{2}$

511

674

522

653

850

600

No

Under Some

No

Under Most

Conditions

No

No

Yes

No

$\mathrm{COS}, \mathrm{CS}_{2}, \mathrm{SO}_{2}$ and $\mathrm{SO}_{3}$

Deactivation of Solvent

Corrosivity of Degradation

Products

Yes

Very

High

High

Yes

No

No

Oxygen Reacts With Solvent

Yes

Very

Requiring Reclaimer

Medium

High

No

Low

Medium

Low

Yes

Low

No 
Contractor Reports Receipt Coordinator Page 5
Technical Progress Report (2nd Semi-Annual) Agreement No. DE-FC21-92MC28178

\section{SOLVENT VERSUS SOUR GAS FLOW RATES}

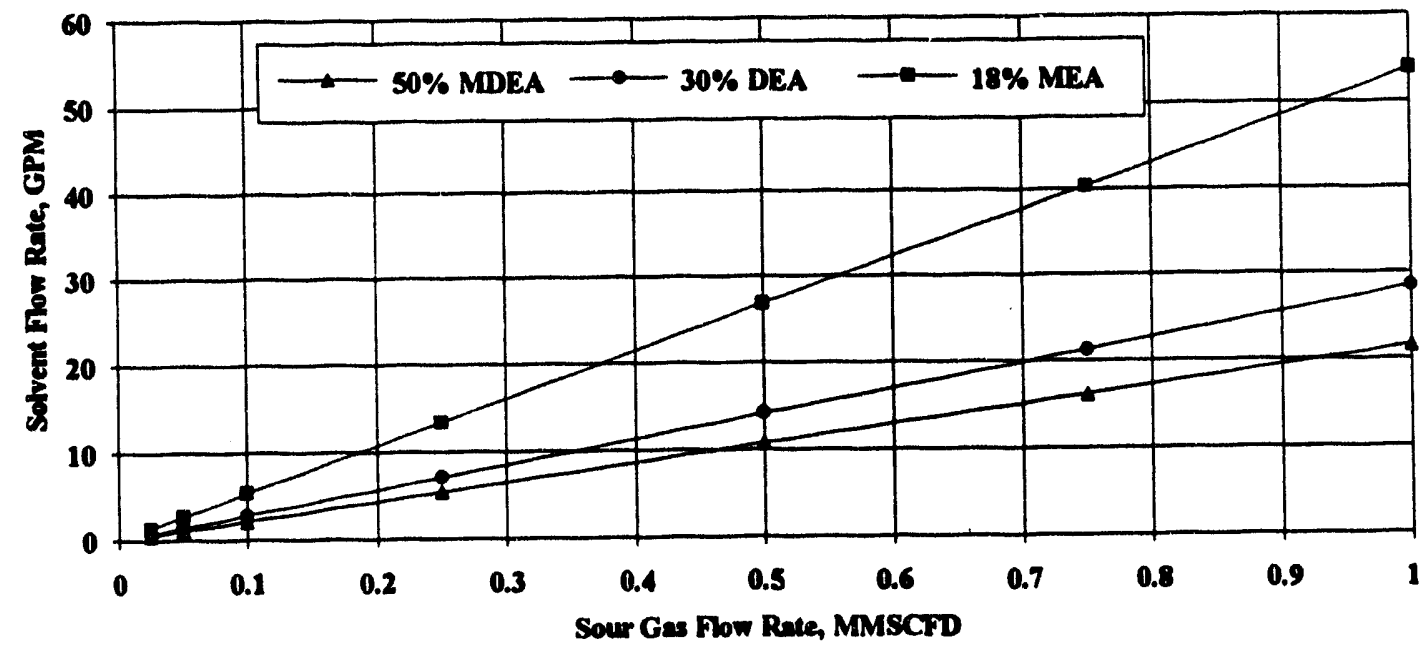

Figure 1. ESTIMATED AMINE SOLVENT FLOWS VERSUS SOUR GAS FLOW FOR A FEED GAS OF $15 \% \mathrm{CO}_{2}$ AND $\mathrm{H}_{2} \mathrm{~S}$ AT 1000 PSIA AND $70^{\circ} \mathrm{F}$

REBOILER DUTY VERSUS SOUR GAS RLOW

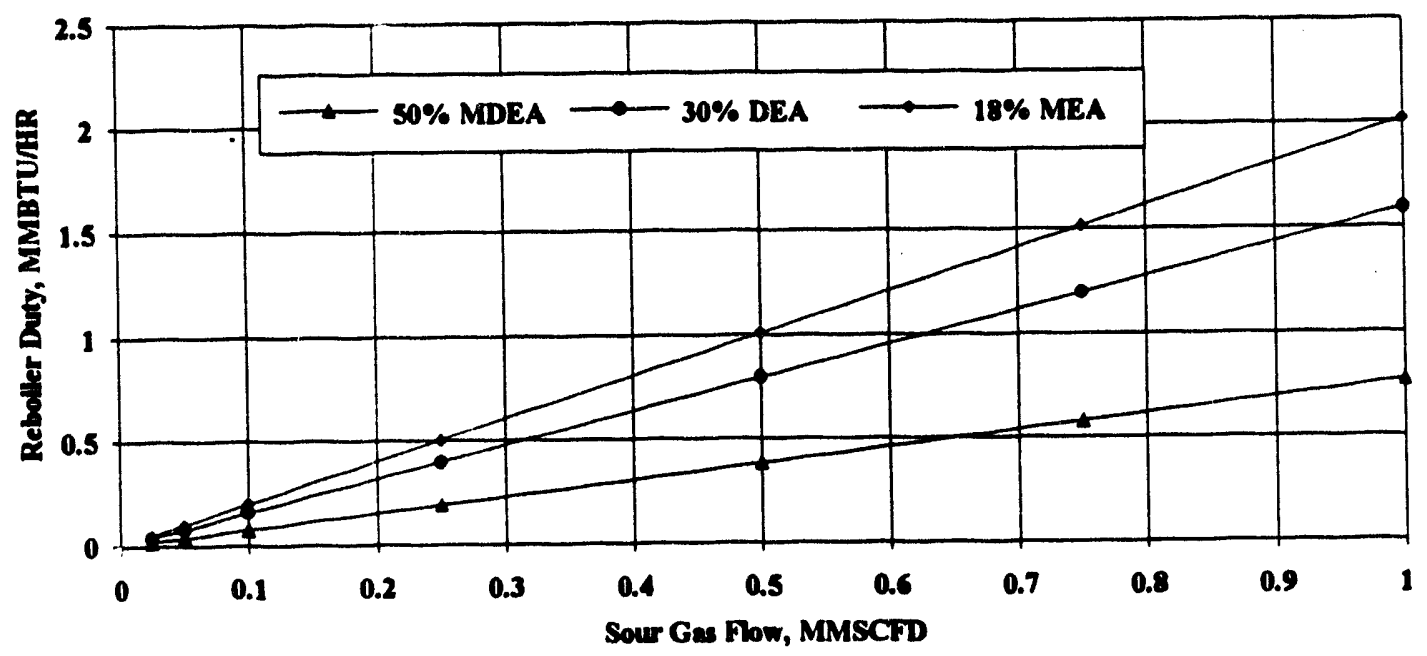

Figure 2. ESTIMATED REBOILER DUTY VERSUS SOUR GAS FLOW FOR A FEED GAS OF $15 \% \mathrm{CO}_{2}$ AND $\mathrm{H}_{2} \mathrm{~S}$ AT 1000 PSIA AND $70^{\circ} \mathrm{F}$

\section{Overall Gas System Design and Construction}

A meeting was held with Mr. Jim Fraizer and Mr. Tom Kresse of Natural Gas Pipeline in

Lombard, Illinois. These gentlemen have had extensive experience in designing gas processing plants. An 
agreement has been put in place where by, at no cost to the project, they will review and comment on the design and construction of the gas processing systems that the China Higee TM unit will be attached to.

Also a consulting agreement is been established with Mr. Jerry Warner to assist in the designing of a gas recycling system to supply mixed gases to this gas processing system. The key components of the recycle system will be the compressors since they will be circulating large volumes of mixed gases (including $\mathrm{CO}_{2}, \mathrm{H}_{2} \mathrm{O}$, and $\mathrm{H}_{2} \mathrm{~S}$ ) at high pressures (from atmospheric to 1,200 psia). Mr. Wamer has been a member of the A.G.A. compressor committee for 8 years and has had 35 years experience in operating and managing underground gas storage facilities and a synthetic gas plant.

Figures 3 and 4 show the current strategies for gas recycle and solvent storage systems being explored. These plans are being used to generate cost estimates. Details of this analysis will be presented in future reports. This information will be summarized and distributed to GRI, DOE, and Glitsch for their review. Plant options with costing estimates for dehydration and acid gas removal have been summarized and will be discussed with GRI, DOE and IGT's Sustaining Membership Program (SMP) during October. The analysis thus far indicates that design and construction of a stationary gas processing plant would not be cost effective when compared to purchasing a skid amine plant.

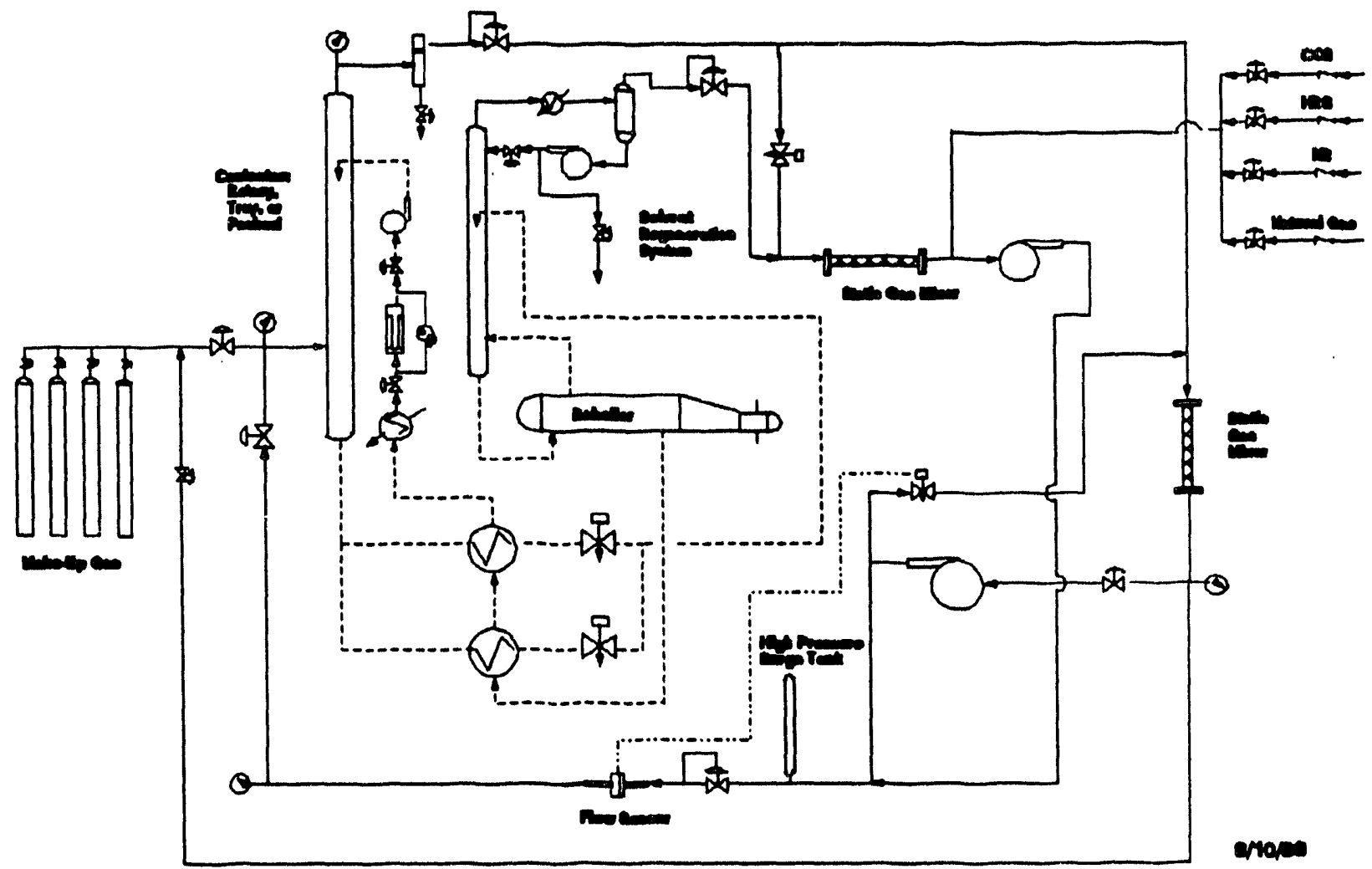

Figure 3. GAS PROCESSING RECYCLE SYSTEM 


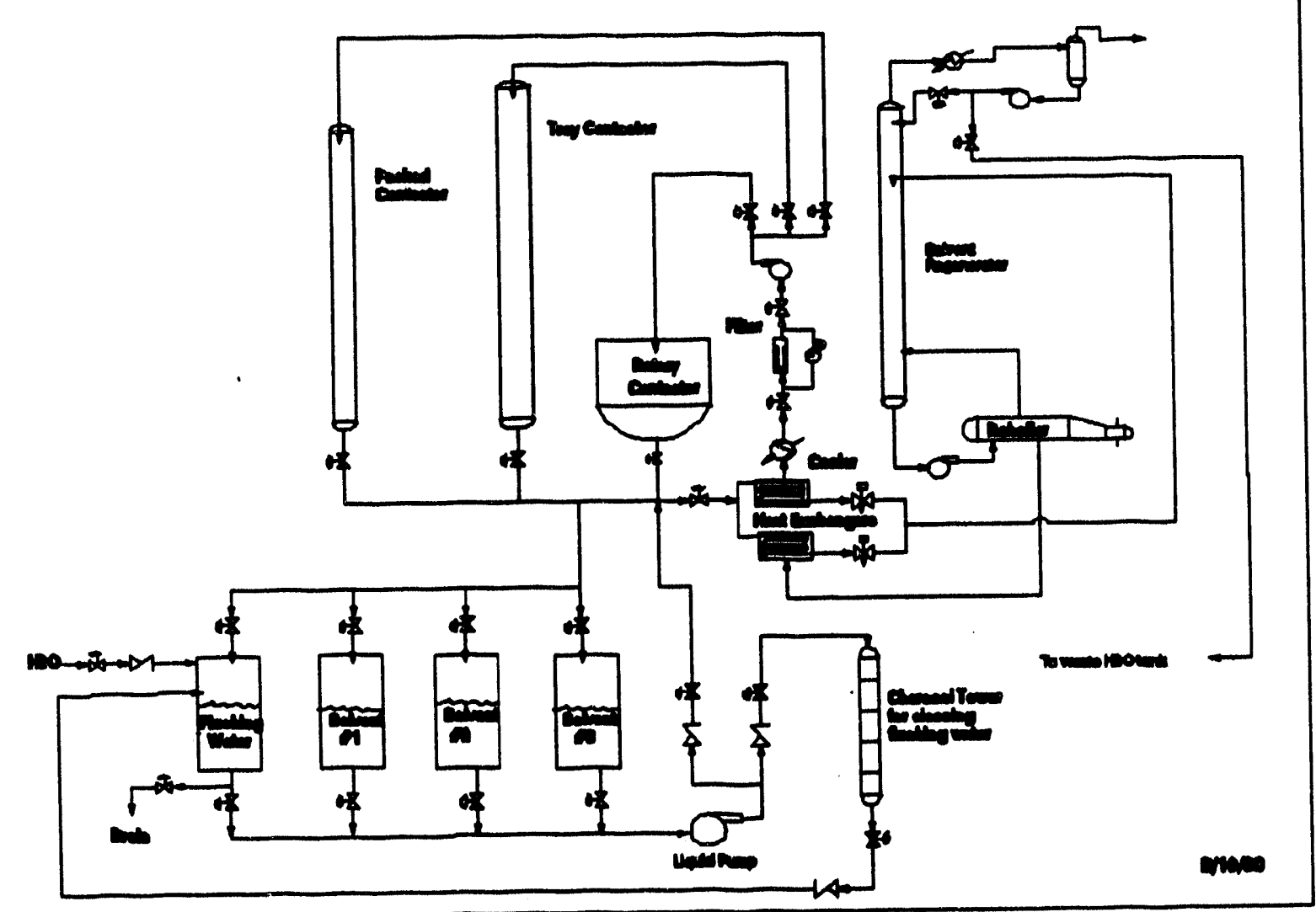

Figure 4. ACID GAS REMOVAL SYSTEM WTTH SOLVENT STORAGE.

\section{Task 8. Field Experimental Studies}

This task was inactive.

\section{Task 9. Data Analysis and Reports}

A stop work order was issued by GRI on this project on February 10th, 1993, pending the results of negotiations between GRI and Glitsch, Inc. In response, IGT limited its efforts on this project to only items that were considered on the critical path and were given specific approval by GRI and DOE. This stop work order was lifted as of June 17th. On June 21, 1993 the following items were sent to Glitsch, Inc. for their review:

- IGT-DOE Contract

- IGT-GRI Contract

- IGT-GRI Licensing Agreement

A consultant/subcontract agreement between IGT and Glitsch concerning the involvement of Paul Platko, Ray Fowler, and Jim Spalding, has also been sent to Glitsch for review. Mr. Platko is an engineer 
at Glitsch with extensive Higee TM operation experience. Mr. Ray Fowler is Glitsch's theoretical expert in Higee TM Technology. Mr. James C. Spalding is the Director of Commercial Affairs at Glitsch and will represent Glitsch's interest, provide licensing and contractual input, and assist in project direction.

A written request for information on the availability of the China Unit was sent to Mr. Spalding of Glitsch on August 11th.

The following action items are still pending:

Glitsch is to provide IGT:

- Royalty-free use of Higee TM Technology.

- No cost loan of the "China Unit".

- Computer and data collection software for the plastic unit.

- Piping diagram of the China Unit.

- Completed subcontract for Mr. Platko, Mr. Fowler, and Mr. Spalding.

During this period the following presentation were made on this project:

- To IGTs SMP Technical Guidance Committee on July 21 st .

- To IGT's SMP Proposal Review Committee on September 15th .

- To IGT's SMP's Review Committee on September 30th .

Presentations planned through the end of 1993 include:

- IGTs SMP semi-annual meeting on October 28th

- DOE's Contractor review meeting on November 16 th.

\section{WORK TO BE PERFORMED NEXT PERIOD}

- Complete all negotiations and processing of agreements.

- Complete assembly, modifications, shakedown, and conduct fluid dynamic studies using the plastic rotary contactor unit.

- Confirmation of project test matrix.

- Finalize dehydration and gas sweetening program strategies.

- Locate, and transport an amine plant and dehydration plant.

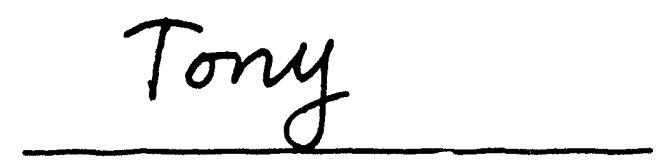

A. L. Lee

Associate Director, Gas Processing and Catalyst Research

312/949-3714

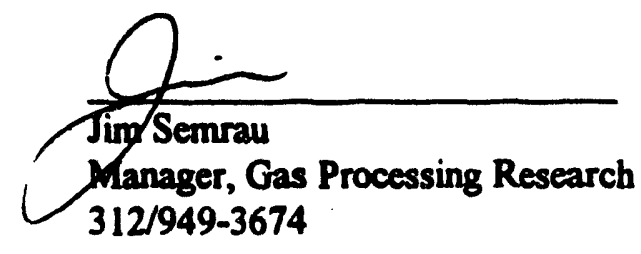


This report was prepared by Institute of Gas Technology pursuant to U.S. Department of Energy Agreement No. DE-FC21-92MC27391 and the Gas Research Institute Contract No. 5092-2222459. However, neither IGT, the Department of Energy, nor GRI, nor any person acting on behalf of any of theni.

a. Makes any warranty or representation, expressed or implied, with respect to the accuracy, completeness, or usefulness of the information contained in this report, or that the use of any information, apparatus, method, or process disclosed in this report may not infringe privately-owned rights, or

b. Assumes any liability with respect to the use of, or for damages resulting from the use of, any information, apparatus, method, or process disclosed in this report.

Reference to trade names or specific commercial products, commodities, or services in this report does not represent or constitute an endorsement, recommendation, or opinion of suitability by GRI or IGT of the specific commercial product, commodity, or service.

This is an interim report; hence, the data, conclusions, and calculations are preliminary and should not be construed as final. 


\section{APPENDDXA}

A-1 
Inputs for calculating amine solvent flows

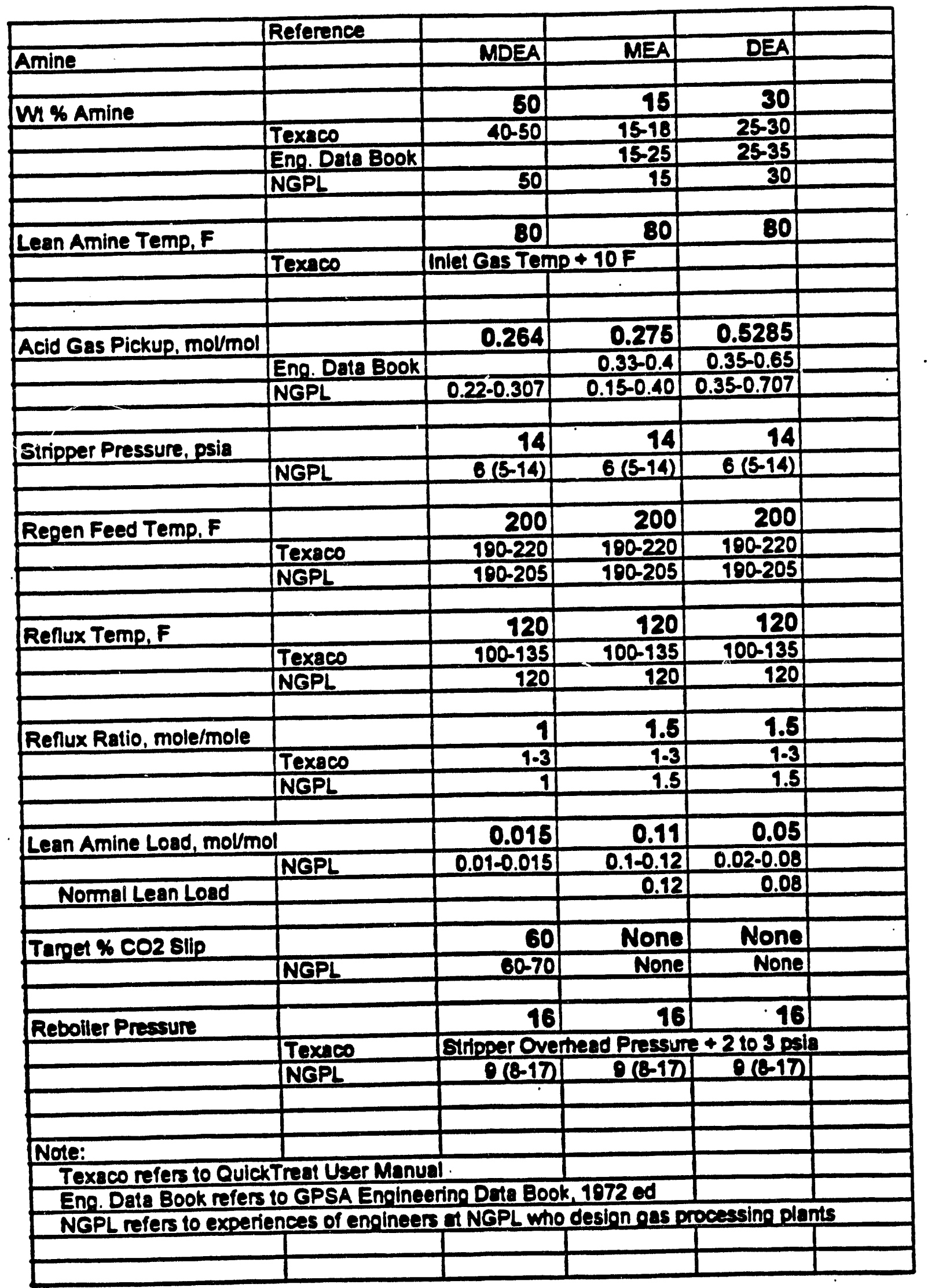


APPENDDX B

B-1 


\section{Bummany of Calcuiations}

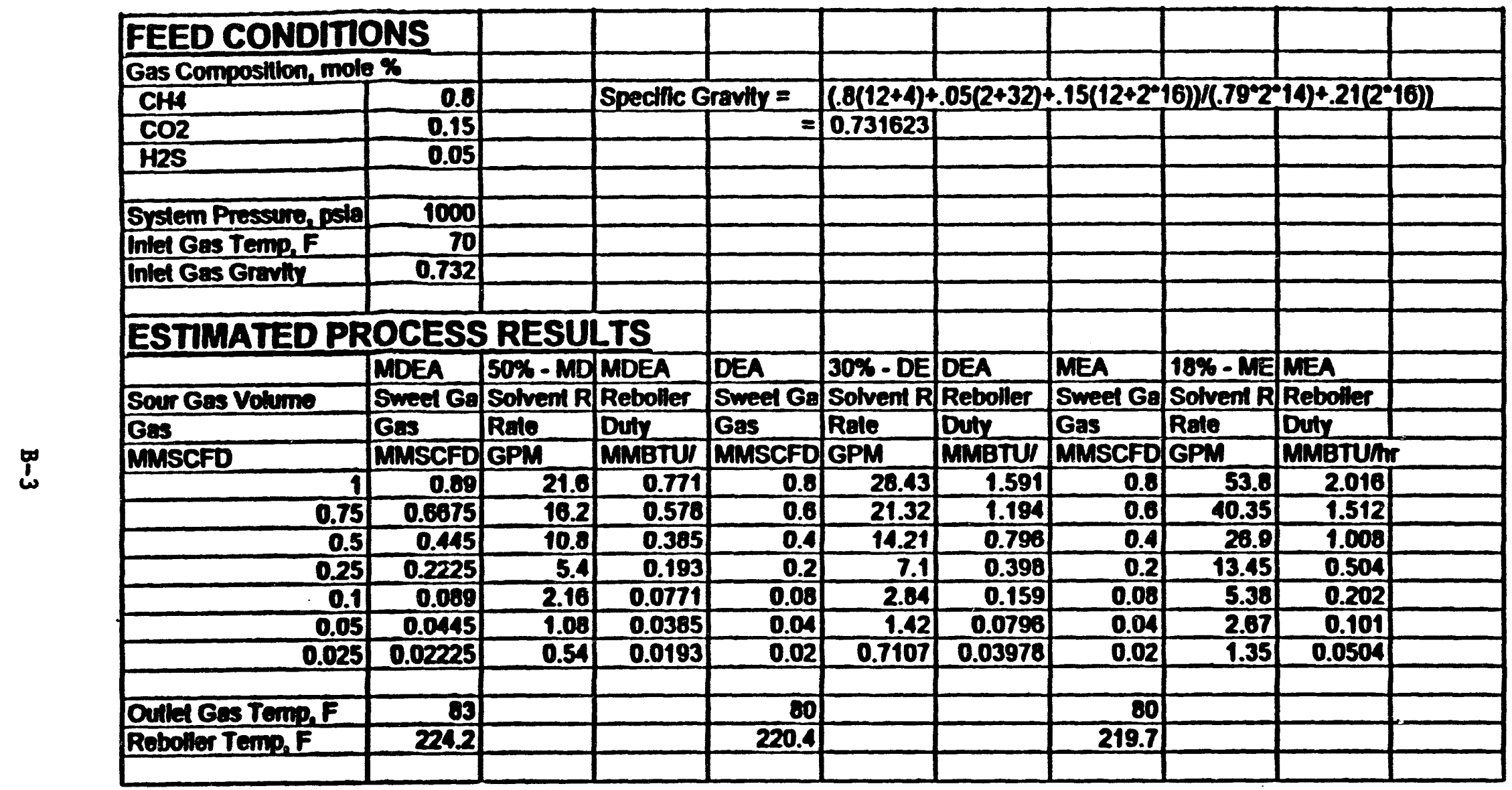


APPENDD C

C-1 
Sour Gas Inlet Data Sour Gas Volume System Pressure Inlet Gas Temperature Inlet Mole t $\mathrm{CO} 2$ Inlet Mole \& IIS Inlet Gas Gravity

$\begin{aligned} \text { (MOMSCFD) } & 1 \\ \text { (PSIA) } & 1000 \\ \text { (Deg F) } & 70 \\ \text { (MOIE \&) } & 15 \\ \text { (MOLE F) } & 5 \\ \text { (AII }=1) & 0.732\end{aligned}$

Ireated Gas outlet Data

Sweet Gas Volume (est) (MMSCFD) 0.89 Outlet Gas Temp

Iean Amine Data

Type of Amine Dtilized

TEXTREAT (MDEA)

Circulation Rate

Weight Percent Amine

Iean Anine Temperature

Iean Amine Ioading

$\begin{aligned} \text { (GPM) } & 21.6068 \\ \text { (Wt. f) } & 50 \\ \text { (Deg F) } & 80 \\ \text { (Mol/Mol) } & 0.015\end{aligned}$

Rich Amine Data Total Acid Gas

Rich Amine Loading

Rich Amine Temp

0.275

103.521

Input Regeneration Data

$\begin{array}{lll}\text { Stripper Overhead Pressure (PSIA) } & 14 \\ \text { Stripper Feed Temperature (Deg F) } & 200 \\ \text { Reflux Temperature } \quad \text { (Deg F) } & 120 \\ \text { Reflux Ratio (Mol ra/mol AG) } & 1 \\ \text { Reboiler Pressure } & \text { (PSIA) } & 16\end{array}$

General Process Temperatures

Regen Overhead Temp

Reboiler Temp

varm Iean Amine Tewp

$\begin{array}{ll}\text { (Deg F) } & 182.345 \\ \text { (Deg F) } & 224.205 \\ \text { (Deg F) } & 126.249\end{array}$

Estimated Dxchanger Duties

\begin{tabular}{|c|c|c|}
\hline $\begin{array}{l}\text { Crose Exchanger Duty } \\
\text { Iean Cooler Duty } \\
\text { Condenser Duty } \\
\text { Reboller Duty }\end{array}$ & $\begin{array}{l}\text { (MABtu/hr) } \\
\text { (MNBEu/hr) } \\
(\text { MNBtu/hr) } \\
(\text { MBBtu/hr) }\end{array}$ & $\begin{array}{l}0.993879 \\
0.43366 \\
0.27015 \\
0.770993\end{array}$ \\
\hline
\end{tabular}

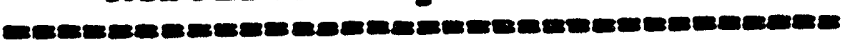

Ihis information is not to be taken as warranty or representation for which we ascume legal reoponoibility. It is offered bolely for your consideration, investigation and verifieation.

THIS PROGRAM Is NOT A RICOROOS EQUIIIBRIUM MODEI. The data presented by these calculations does NOT GUARANIEE that the treated gas will

meet the required product specifications nor that any requested level of $\mathrm{CO} 2$ ilppage can be achieved. please contact your IrXxaco ctimicaI representative for actual equilibrium verification. 
Sour Gas Inlet Data Sour Gas Volume System Pressure Inlet Gas Temperature Inlet Mole \& $\mathrm{CO}_{2}$ Inlet Mole \& H2S Inlet Gas Gravity

$\begin{aligned} \text { (MMSCFD) } & 0.75 \\ \text { (PSIA) } & 1000 \\ \text { (Deg F) } & 70 \\ \text { (Mole t) } & 15 \\ \text { (Mole t) } & 5 \\ \text { (Adr } 1 \text { ) } & 0.732\end{aligned}$

Ireated Gas Outlet Data

Sweet Gas Volume (est) (MMSCFD) 0.6675 Outlet Gas Temp

- 002 in Sweet Target SIippage

60

cos in sweet, NOT guaranteed (Mole \&)

10.1224

Iean Amine Data

Iype of Amine Utilized

TEXTREAT

Circulation Rate

(GPM)

Weight Percent Amine

Iean Amine Temperature

(WE. \&)

(Deg F)

Lean Amine Loading

(Mol/Mol)

16.2051

50

80

0.015

Iich Amine Data

Total Acid Gas

Rich Amine Loading

Rich Amine Temp

\author{
(Moles/hr) \\ (Mol/Mol) \\ (Deg F)
}

9.0255

0.275

203.521

Iropat Regeneration Data

stripper overhead Pressure (PSIA)

14

Stripper Feed Temperature (Deg F)

200

Reflux Temperature

(Deg F)

Reflux Ratio

(Mol H2O/mol AG)

Reboiler Pressure

(PSIA)

120

1

16

Eeneral Process Temperatures

Regen Overhead Temp

Reboiler Temp

Warm Iear Amine Temp

(Deg F)

182.345

(Deg F)

224.205

(Deg F)

126.249

Irtimated Exchanger Duties

Cross Exchanger Duty

Iean Cooler Duty

Condeneer Duty

Reboiler Duty

(MNBtu/br)

(MABtu/br)

0.745409

0.325245

(MDBEu/br)

0.202612

(MarBtu/br)

0.578245

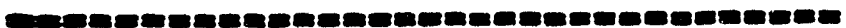

Tris Information is not to be taken as warranty or representation for which we assume legal reoponoibility. It is offered solely for your consideration, investigation and verification.

IHIS PROGRAM IS NOT A RIGOROUS EQOILIBRIUM MODEL. The data presented by these calculations does NOT GUARANTEE that the treated gas will

meet the required product opecifications nor that any requested level of CO2 slippage can be achieved. Please contact your TEXACO CHIMICAI representative for actual equilibrium verification. 
Soor Gas Inlet Data

Sour Gas Volume

System Pressure

Inlet Gas Temperature

Inlet Mole \& $\mathrm{CO} 2$

Inlet Mole H2S

(MMSCFD)

(PSIA)

(Deg F)

(Mole \&)

(Mole t)

(AII = 1)

Inlet Gas Gravity

Ireated Gas outlet Data

Sweet Gas Volume (est) (MuscFD)

Outlet Gas Temp

- 202 in Sweet Target silppage.

(Deg F)

co2 in (Mole \&)

Iean Amine Data

Iype of Amine Dtilized

Circulation Rate

Weight Percent Amine

Iean Amine Temperature

Iean Amine Loading

Fich Amine Data

Total Acid Gas

Rich Amine Ioading

Rich Amine Temp

Irorut Regeneration Data

Stripper Overhead Pressure (PSIA)

Stripper Feed Temperature (Deg F)

RefIux Temperature

Reflux Ratio

Reboiler Pressure

(Mol H2O/mol AG)

(PSIA)

(GPM)

(Wt. \&)

(Deg F)

(MOL/MOL)

(Moles/hr)

(Mol/Mol)

(Deg F)

0.5

1000

70

15

5

0.732

0.445

82.9255

60

20.124

TEXTREAT

10.8034

50

80

0.015

6.017

0.275

103.521
14

200

120

1

16

Seneral Process Temperatures

Regen Overhead Temp

Reboiler Temp

Warm Iean Arine Temp

(Deg F)

(Deg F)

(Deg F)

Istimated Exchanger Duties

Cross Exchanger Duty
Iean Coolex Duty
Condeneer Duty
Rebojler Duty

(MABta/hr)

(MNBCu/br)

(MABEu/br)

(MBEu/br)

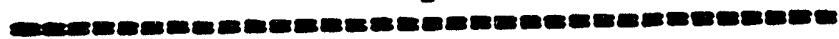

182.345

224.205

126.249

This Information is not to be taken as warranty or representation for which we assume legal responsibility. It lo offered solely for your consideration, investigation and verification.

THIS PROGRAM IS NOT A RICOROOS EOUIIIBRIOM NODEL. The data presented by these calculations does NOT GUARANILE that the treated gas will meet the required product specifications nor that any reguested level of 02 slippage cas be achieved. please contact your Taxaco crismICAI representative for actual equilibrium verification. 
Sour Gas Inlet Data Sour Gas Volume System Pressure Inlet Gas Temperature Inlet Mole \& $\mathrm{CO} 2$ Inlet Mole of H2S Inlet Gas Gravity

$\begin{aligned} \text { (MSCFD) } & 0.25 \\ \text { (PSIA) } & 1000 \\ \text { (Deg F) } & 70 \\ \text { (MOIE } & 15 \\ \text { (Mole } 1) & 5 \\ \text { (AIr } 1 \text { ) } & 0.732\end{aligned}$

Ireated Gas outlet Data

Bweet Gas Volume (est) ( (MuSCFD)

Outlet Gas Temp

(Deg F)

- 02 in Sweet Target S1ippage

CO2 in sweet. NOT guaranteed (Mole t)

0.2225

82.9255

60

10.1124

Ien Amine Data

Iype of Amine Otilized

\section{TEXIXREAT}

Circulation Rate

Weight Percent Amine

Iean Amine Temperature

Iean Amine Loading

$\begin{aligned} \text { (GPM) } & 5.40169 \\ \text { (Wt. F) } & 50 \\ \text { (Deg F) } & 80 \\ \text { Mol/MOI) } & 0.015\end{aligned}$

Rich Amine Data

Total Acid Gas

Rich Amine Loading

Rich Amine Temp

$\begin{aligned} \text { (Moles/hr) } & 3.0085 \\ \text { (Mol/Mol) } & 0.275 \\ \text { (Deg F) } & 103.521\end{aligned}$

Input Regeneration Data

$\begin{array}{lll}\text { Stripper Overhead Pressure (PSIA) } & 14 \\ \text { Stripper Feed Temperature (Deg F) } & 200 \\ \text { Reflux Temperature } & \text { (Deg F) } & 120 \\ \text { Reflux Ratio (Mol H20/mol AG) } & 1 \\ \text { Reboiler Pressure } & \text { (PSIA) } & 16\end{array}$

General Process Temperatures

Regen Overhead Temp

Reboiler Temp

Varm Iean Amise Temp

(Deg F)

(Deg F)

(Deg F)

Eotimated Exchanger Duties

Crose Exchanger Duty (MABtu/hr)

Iean Cooler Duty

Condenser Duty

Reboiler Duty

(MDBEu/hr)

(MOBEu/hr)

(MDBEu/hr)
182.345

224.205

126.249

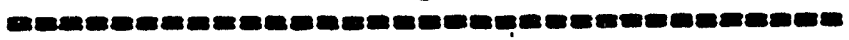

0.24847
0.108415
0.0675375
0.192748

This information is not to be taken as warranty or representation for which we assume legal responsibility. It is offered solely for your consideration, investigation and verification.

IHIS PROGRAM IS NOT A RICOROUS EQUILIBRIOM MODEL. The data preBented by these calculations does NOT GUARANIJE that the treated gas will meet the reguired product specifications nor that any requested level of $\mathrm{CO} 2$ ilippage can be achieved. Please contact your IEXACO CHMICAI representative for actual equilibrium verification. 
Sour Gas Inlet Data

Sour Gas Volume

System Pressure

Inlet Gas Temperature

Inlet Mole of $\mathrm{CO} 2$

Inlet Mole \& 1125

Inlet Gas Gravity

$\begin{aligned} \text { (MMSCFD) } & 0.1 \\ \text { (PSIA) } & 1000 \\ \text { (Deg F) } & 70 \\ \text { (MoIE \&) } & 15 \\ \text { (Mole \&) } & 5 \\ \text { (Aix - 1) } & 0.732\end{aligned}$

Treated Gas Outlet Data

Sweet Gas Volume (est) (MSCFD) 0.089

Outlet Gas Temp

(Deg F)

- 02 in Sweet Target SIippage

Co2 in Sweet. NOT guaranteed (Mole t)

82.9255

60

10.124

Iean Amine Data

Type of Amine Utilized

IESTRRAAT

Circulation Rate

(GPM)

Weight Percent Amine

Iean Amine Temperature

Iean Anine Loading

(wt. \&)

(Deg F)

(Mol/Mol)

2.16068

50

80

0.015

Rich Anine Data

Total Acid Gas

Rich Amine Ioading

Rich Amine Temp

(Moles/hr)

(Mol/Mol)

(Deg E)

Input Regeneration Data

Stripper overhead Pressure (PSIA)

Stripper Feed Temperature (Deg F)

Reflux Temperature

Reflux Ratio

(Deg F)

(Mol H20/mol AG)

Reboiler Pressure

(PSIA)

General Process Temperatures

Regen Overhead Temp

reboiler Temp

varm Iean Amide Temp

(Deg F)

(Deg F)

(Deg F)

Estimated Exchanger Duties

Cross Exchanger Duty (MiBtu/br)

Iean Coolex Duty

Condenser Duty

Reboiler Duty

(MNBEu/br)

(MDBtu/br)

(MABEu/hr)
1.2034

0.275

103.521

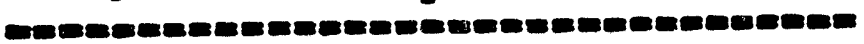

14

200

120

1

16

This Information is not to be taken as warranty or representation for which we aseume legal responsibility. It is offered solely for your consideration, investigation and verification.

182.345

224.205

126.249

IHIS PROGRAM IS NOT A RICOROUS EOUILIBRIOM MODEL. The data presented by these calculations does NOT GUARANIEE that the treated gas will

meet the required product opecificationo nor that any requested level

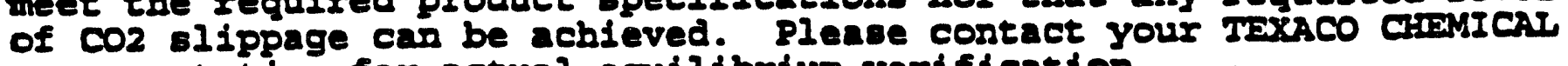
representative for actual equilibrium verification. 
Sour Gas Inlet Data Sour Gas Volume System Preseure Inlet Gas Temperature Inlet Mole $\mathrm{CO} 2$ Inlet Mole of I2S Inlet Gas Gravity

$\begin{aligned} \text { (MNSCFD) } & 0.05 \\ \text { (PSIA) } & 1000 \\ \text { (Deg F) } & 70 \\ \text { (Mole \&) } & 15 \\ \text { (Mole t) } & 5 \\ \text { (AIY }=1) & 0.732\end{aligned}$

Ireated Cas outlet Data

sweet Gas Volume (est) (MuSCFD) 0.0445

Outlet Gas Temp (Deg F)

- 002 In Eweet Target SIippage (t)

co2 in sweet. NOT guaranteed (Mole \&)

82.9255

60

10.1124

Iean. Amine Data

Iype of Amine Vtilized

TEXIREAT

Circulation Rate

Weight Percent Amine

Iean Amine Temperature

Iean Amine Ioading

(GPM)

(Wt. *)

(Deg F)

(Mol/Mol)

1.08034

50

80

0.015

Rich Amine Data

Total Acid Gas

Rich Amine Loading

Rich Amine Temp

(Moles/hr)

(MOl/Mol)

(Deg F)
0.6017

0.275

103.521

Impor Regeneration Data Stripper Overhead Pressure (PSIA) Stripper Feed Temperature (Deg F) 200 Refiux Temperature Reflux Ratio (Deg F) Reboiler Pressure (MOI $120 / \mathrm{mol}$ AG) (PSIA)

120

1

16

General Process Temperatures Regen Overhead Temp Reboiler Temp varm Iean Amine Temp

$\begin{array}{ll}\text { (Deg F) } & 182.345 \\ \text { (Deg F) } & 224.205 \\ \text { (Deg F) } & 126.249\end{array}$

Zutimated Exebanger Duties

Cross Exchanger Duty (MaBtu/hr) 0.049694

Iean Coolex Duty

(MASBEu/br)

0.021683

Condenser Duty

(MNBEu/hr)

Reboiler Duty

(MDBtu/br)

0.0135075

0.0385496

This information is not to be taken as warranty or representation for which we assume legal responsibility. It is offered solely for your consideration, investigation and verification.

IHIS PROCRAM IS NOT A RICOROUS EOUIIIBRIUM MODEL. The data preBented by these calculations does NOT GUARANIEE that the treated gas will meet the required product specifications nor that any requested level of $\mathrm{CO} 2$ slippage can be achieved. please contact your IrXXACO CHMICAI representative for actual equilibrium verification. 
Eow Gas Inlet Data

Sour Gas Volume

System Pressure

Inlet Gas Temperature

Inlet Mole \& $\mathrm{CO} 2$

Inlet Mole 825

Ialet Gas Gravity

$\begin{aligned} \text { (MMSCFD) } & 0.025 \\ \text { (PSIA) } & 1000 \\ \text { (Deg F) } & 70 \\ \text { (Mole f) } & 15 \\ \text { (Mole }) & 5 \\ \text { (Air }=1) & 0.732\end{aligned}$

Ireated Gas Outlet Data

Sweet Gas Volume (Cst) (MMSCFD) 0.02225

Outlet Gas Temp

(Deg F)

- 022 in Sweet Target slippage

002 in sweet. NOT guaranteed (Mole t)

82.9255

60

10.1224

Inan Amine Data

Iype of Amine Utilized

TEXIREAT

Circulation Rate

Weight Percent Anine

Iean Amine Temperature

Iean Amine Loading

(GPM)

(Wt. \&)

(Deg F)

(Mol/Mol)

(Moles/hr)

(Mol/Mol)

(Deg F)

Rich Amine Temp

Iotal Acid Gas
Rich Anine Loading

Rich Amine Data

ach sulase Tamp

Inowt Regeneration Data

Stripper Overhead Pressure (PSIA)

Stripper Feed Temperature (Deg F) 200

Reflux Temperature (Deg F)

RefIux Ratio (Mol z20/mol AG)

Reboiler Pressure (PSIA)

120

1

16

General Process Temperatures

$\begin{array}{lll}\text { Regen Overhead Teup } & \text { (Deg F) } & 182.345 \\ \text { Reboiler Temp } & \text { (Deg F) } & 224.205 \\ \text { Varm Iean Amine Teup } & \text { (Deg F) } & 126.249\end{array}$

Eximated Exchanger Duties

$\begin{array}{lll}\text { Crose Exchanger Duty } & \text { (MBtu/hr) } & 0.024847 \\ \text { Ieas Copler Duty } & \text { (MNBtu/hr) } & 0.0108415 \\ \text { Condenser Duty } & \text { (MBtu/hr) } & 0.00675375 \\ \text { Reboiler Duty } & \text { (MBDu/hr) } & 0.0192748\end{array}$

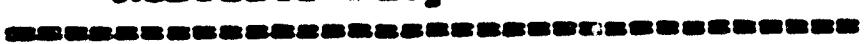

Inis information is not to be taken a warranty or representation for which we assume legal responsibility. It is offered solely for your consideration. investigation and verification.

IHIS PROGRAM IS NOT A RICOROOS EQDILIBRIUM MODEL. The data presented by these calculations does NOT GUARANTEs that the treated gas will meet the required product opecification nor that any requested level of CO2 Blippage can be achieved. please contact your IEXACO craMICAI representative for actual equilibrium verification. 
Souss Gas Inilet Data

Sour Gas Volume

System Pressure

Inlet Gas Temperature

Inlet Mole $\mathrm{CO} 2$

Inlet Mole 825

Inlet Gas Gravity

$\begin{aligned} \text { (MNSCFD) } & 0.024 \\ \text { (PSIA) } & 1000 \\ \text { (Deg F) } & 70 \\ \text { (MoIE }) & 15 \\ \text { (Mole ) } & 5 \\ \text { (Aix }=1) & 0.732\end{aligned}$

Irented Gas Outlet Data

Sweet Gas volume (est) (MascFD) 0.02136

Outlet Gas Temp

- co2 in Sweet Target s1ippage

(Deg F)

(\$)

co2 is sweet. NOT guaranteed (Mole t)

82.9255

60

10.2124

Ion Amine Data

zype of Amine otilized

TEXIREAT

Circulation Rate

(GPM)

Weight Percent Amine

Iean Amine Temperature

(Wt. \&)

(Deg F)

Iean Anine Loading

(MOI/MOL)

0.518562

50

80

0.015

Rich Amine Data

Total Acid Gas

Rich Amine Ioading

Rich Amine Temp

(Moles/hr)

(Mol/Mol)

(Deg F)

0.288816

0.275

103.521

Inout Regeneration Data

Stripper Overhead Pressure (PSIA) 14

Stripper Feed Temperature (Deg F) 200

Reflux Temperature (Deg F) 120

Reflux Ratio (Mol H20/mol AG) 1

Reboiler Pressure $\quad$ (PSIA) 16

Eeneral Process Temperatures

Regen Overhead Temp (Deg F) 182.345

Rebolier Temp

varm Lean Amine Temp

(Deg F)

224.205

(Deg E) 126.249

Ixtimated Exchanger Dutieo

$\begin{array}{lll}\text { Crose Dxchanger Duty } & \text { (MBtu/hr) } & 0.0238531 \\ \text { Iean Cooler Duty } & \text { (MBtu/hr) } & 0.0104079 \\ \text { Condenser Duty } & \text { (MBtu/hr) } & 0.0064836 \\ \text { Reboiler Duty } & \text { (MBtu/br) } & 0.0185038\end{array}$

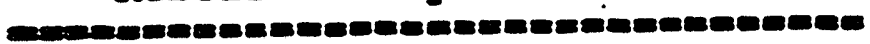

This information is not to be taken as warranty or representation for which we assume legal responsibility. It is offered solely for your consideration, investigation and verification.

IHIS PROGRAY IS NOT A RICOROUS EQUIIIBRIUM MODEL. The data presented by these calculations does NOT GWARANIIE that the treated gas will meet the required product pecifications nor that any requested Ievel of $\mathrm{CO} 2$ slippage can be achieved. please contact your IEXACO CHEMICAI representative for actual equilibrium verification. 
Sowr Gas Inlet Data Sour Gas Volume System Pressure Inlet Gas Temperature Inlet Mole \& $\mathrm{CO} 2$ Inlet Mole H2S Inlet Gas Gravity

$\begin{aligned} \text { (MMSCFD) } & 1 \\ \text { (DSIA) } & 1000 \\ \text { (DEg F) } & 70 \\ \text { (MOIE \&) } & 15 \\ \text { (MOIE t) } & 5 \\ \text { (AII }=1) & 0.732\end{aligned}$

Ireated Gas Outlet Data

$\begin{array}{lll}\text { Sweet Gas Volume (est) (MSCFD) } & 0.8\end{array}$ outlet Gas Temp

(Deg F) 80

Iean Amine Data

Iype of Amine Utilized

Circulation Rate

Weight Percent Amine

Iean Amine Temperature

Iean Amine Loading

(GPM)

(Wt. \&)

(Deg F)

(Mol/Mol)

Rich Amine Data

Total Acid Gas

Rich Amine Loading

Rich Amine Temp
(Moles/hr)

(Mol/Mol)

(Deg F)
MEA

53.8067

18

80

0.11

21.88

0.385

104.288

Inove Regeneration Data Stripper Overhead Pressure (PSIA) Stripper Feed Temperature (Deg F) Reflux Temperature (Deg F) Reflux Ratio (Mol H2O/mol AG) Reboiler Pressure

(PSIA)

14

200

120

1.5

16

188.96

219.699

221.624

General Process Temperatures

Regen Overhead Temp

Reboiler Temp

Warm Lear Amine Temp

Estimated Exchanger Duties

Cross Exchanger Duty

Iean Cooler Duty Condenser Duty

Reboiler Duty
(Deg F)

(Deg F)

(Deg F)
(MMBEu/br)

(MNBEu/br)

(MABEu/br)

(MABEu/br)

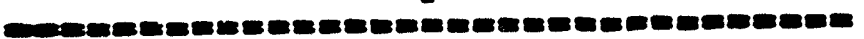

This information 18 not to be taken as warranty or representation for which we assume legal responsibility. It is offered colely for your consideration, investigation and verification.

2.67998

1.20713

0.73371

2.01579

IEIS PROGRAM IS NOT A RICOROOS EOUIIIBRIOM MODXI. The data preBented by these calculations does NOT GUARANISE that the treated gas w111 meet the regrifired product opeciflcatlons nor that any reguested level of $\mathrm{CO} 2$ slippage can be achieved. please contact your IEXACO CHMICAI representative for actual equilibrium verification. 


\section{Quicktreat (tm) Basic Calculations}

Estimated Performance

Bowr Gas Inlet Data Soux Gas Volume Syatem Preseure Ir iet Gas Temperature Inlet Mole $\mathrm{CO} 2$ Inlet Mole 825 Inlet Gas Oravity

$\begin{aligned} \text { (MASCD) } & 0.75 \\ \text { (DSIA) } & 1000 \\ \text { (Deg F) } & 70 \\ \text { (Mole \&) } & 15 \\ \text { (Mole \&) } & 5 \\ \text { (AIr } 1 \text { ) } & 0.732\end{aligned}$

Ireated Cas Outlet Data Sweet Gas Volume (est) (MuscTD) 0.6 Outilet Gas Temp

Iens Amine Data Iype of Amine Dt111zed

MISA

Circulation Rate Weight Percent Amine Iean Amine Temperature Iean Anine Ioading

(GPM)

(Wt. \&)

(Deg F)

(MOL/MOL)

(Moles/hr)

(Mol/Mol)

(Deg F)
Total Asid Gas

Rich Amine Ioading

Rich Amige Temp

Imput Regeneration Dara

Stripper Overhead Pressure (PSIA) 14

Strippex Feed Temperature (Deg F)

Reflux Temperature

(Deg F)

Reflux Ratio

(Mol 120/mol AG)

Reboiler Preseure

(PSIA)

40.355

18

80

0.11

16.41

0.385

104.288

200

220

1.5

26

Ceneral Process Temperaturen

Regen Overhead Temp

Reboiler Temp

(Deg F)

(Deg F)

188.96

Tarm Ieas Amine Temp

(Deg F)

219.699

121.624

Ertimated Exchanger Duties

Crose Exchanger Duty

Iean Cooler Duty

Condenser Duty

Reboiler Duty

(MABEL/br)

(MDBEu/br)

(MDBEu/br)

(MDEtu/br)

-

2.00598

0.830351

0.550283

2.51284

This information is not to be taken as warranty or representation for which we ascume legal responsibility. It is offered solely for your consideration, investigation and verifiention.

IHIS PROGRAM IS NOT A RICOROUS EOUILIBRIDM MODEI. The data presented by these calculation does NOT GUARANIEE that the treated gas will

meet the required product apeciflcation nor that any reguested level of $\mathrm{CO2}$ elippage can be achleved. please contact your Irxaco CrimicAl representative for actual equilibrium verification. 
Sour Gas Inlet Data Sour Gas Volume System Pressure Inlet Gas Temperature Inlet Mole $\mathrm{CO} 2$ Inlet Mole \& 128 Inlet Gas Gravity

$\begin{aligned} \text { (MASCFD) } & 0.5 \\ \text { (PSIA) } & 1000 \\ \text { (Deg F) } & 70 \\ \text { (MoIE }) & 15 \\ \text { (Mole }) & 5 \\ \text { (Air }=1 \text { ) } & 0.732\end{aligned}$

Treated Gas Outlet Data

$\begin{array}{lll}\text { Sweet Gas Volume (est) } & \text { (MMSCFD) } & 0.4 \\ \text { Outlet Gas Temp } & \text { (Deg F) } & 80\end{array}$

Ieno Amine Data

Type of Amine otilized

MISA

Circulation Rate

Weight Percent Amine

Iean Amise Temperature

Iean Amine Ioading

(GPM)

(Wt. \&)

(Deg F)

(MOL/MOL)

26.9033

18

80

0.11

Rich Amine Data

Total Acid Gas

Rich Anine Ioading

(Moles/hr)

(Mol/Mol)

20.94

Rich Amine Temp

(Deg F)

0.385

204.288

Imput Regeneration Data

$\begin{array}{ll}\text { Stripper Overhead Prescure (PSIA) } & 14 \\ \text { Stripper Feed Temperature (Deg F) } & 200\end{array}$

Reflux Temperature

(Deg F) 120

ReElux Ratio

(Mol 820/mol AG)

2.5

Reboiler Preseure

(PSIA) $\quad 16$

reneral Process Temperatures

Regen Overhead Temp (Deg F) 188.96

Rebolier Temp

(Deg F)

219.699

varm Iean Amine Temp

(Deg F)

121.624

Drtimated Exchanger Duties

Crose Exchanger Duty (NaBtu/br)

Iean Cooler Duty

Condencer Duty

Reboilex Duty

(MDEtu/br)

(MABtu/br)

(MBBta/br)

1.33999

0.553567

0.366855

1.00789

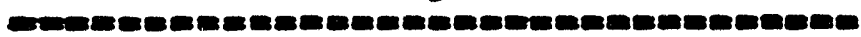

This information is not to be taken as warraty or repreaentation for which we asoume legal renponsibility. It is offered bolely for your consideration, investigation and verification.

IHIS PROCRAY IS FOT A RICOROOS EOUIIIBRIUM MODEH. The data presented by these calculations does NOT GUARANTEs that the treated gas will meet the required product opecifications nor that any reguested level of 022 ilppage can be achleved. please contact your Ixxaco cranical representative for actual equilibrium verification. 
Sour Cas Inlet Data Sour Gas Volume Syetem Pressure Inlet Gas Temperature

Inlet Mole $\mathrm{CO2}$

Inlet Mole 525

Inlet Gas Gravity

$\begin{aligned} \text { (MISCFD) } & 0.25 \\ \text { (PSIA) } & 1000 \\ \text { (Deg F) } & 70 \\ \text { (MOIE \&) } & 15 \\ \text { (MOIE F) } & 5 \\ \text { (AIr } 1 \text { ) } & 0.732\end{aligned}$

Ireated Gas Outlet Data

Sweet Gas Volume fest) (MuscFD) 0.2

Outlet Gas Temp

(Deg F) 80

Iens Amine Data

Iype of Anine Utilized

Iris:

Circulation Rate

Weight Percent Amine

Iean Amine Temperature

Iean Amine Loading

(GPM)

(Wt. \&)

(Deg F)

(Mol/Mol)

(Moles/hr)

(Mol/Mol)

(Deg F)

Rich Amine Temp

Imput Regeneration Data

Stripper Overhead Pressure (PSIA)

Stripper Feed Temperature (Deg F)

Reflux Temperature

Reflux Ratio

(Deg F)

(Mol H20/mol AG)

Reboiler Pressure

(PSIA)

General Process Temperatures

Regen Overhead Temp

Reboilex Temp

Warm Iean Amine Temp

(Deg F)
(Deg F)
(Deg F)

Estimated Exchanger Duties

Crose Exchanger Duty

Iean Cooler Duty

Condeneer Duty

Reboller Daty

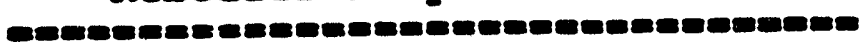

(MDBtu/hr)

(MDBtu/br)

(MDBtu/hr)

(MDBtu/hr)
13.4517

18

80

0.11
5.47

0.385

104.288
188.96

219.699

121.624

14

200

120

1.5

16

0.669995

0.276784

0.183428

0.503947

This information is not to be taken as warranty or representation for which we ascume legal responsibility. It is offered solely for your connideration, inventigation and veriflention.

IHIS PROGRAM IS NOT A RICOROOS EOUIIIBRIUM MODEL. The data prenented by these calculation does NOT GUARANIras that the treated gas will meet the required product opeciflcations nor that any requested level of $\mathrm{CO} 2$ slippage can be achieved. please contact your IBXACO CimMICAI representative for actual equilibrium verification. 


\section{Quicktreat (tm) Basic Calculations}

Estimated Performance

Sowr Gas Inlet Data Sour Gas Volume System Pressure Inlet Gas Temperature Inlet Mole \& $\mathrm{CO} 2$

Inlet Mole 425 Inlet Gas Gravity

$\begin{aligned} \text { (MMSCFD) } & 0.1 \\ \text { (PSIA) } & 1000 \\ \text { (INeg F) } & 70 \\ \text { (MCIE \&) } & 15 \\ \text { (Mole \&) } & 5 \\ \text { (Air - I) } & 0.732 \\ & \\ \text { (MNSED) } & 0.08 \\ \text { (Deg F) } & 80\end{aligned}$

Iean Amine Data

Type of arine Dtilized

MISA

Circulation Rate (GPM)

weight Percent Amine

(Wt. \&)

Iean Amine Temperature

(Deg F)

Iean Amine Ioading

18

80

0.11

Rich Amine Data
Total Acid Gas
Rich Amine Ioading
Rich Amine Temp

(Moles/hr)

(Mol/Mol)

(Deg F)
2.188

0.385

104.288

Impat Regeneration Data

Stripper Overhead Pressure (PSIA)

Stripper Feed Temperature (Deg F) 200

Reflux Temperature (Deg F) 120

Refiux Ratio (Mol H2O/mol AG) 1.5

Reboiler Pressure $\quad 16$

Eeneral Process Temperatures

Regen Overhead Temp (Deg F) 188.96

Reboiler Temp

Varm Iean Anine Temp

(Deg F)

219.699

(Deg F)

121.624

Extimated Exchanger Duties

Crose Exchanger Duty

Iean Cooler Duty

(MDBEu/hr)

0.267998

Condenser Duty

Reboiler Duty

(MBBtu/hr)

0.120713

(MDBBeu/br)

0.073371

(MNBtu/hr)

0.201579

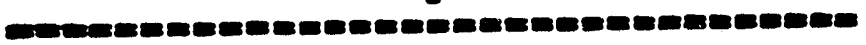

This information is not to be taken as warranty or representation for which we aseume legal responsibility. It is offered bolely for your consideration, investigation and verification.

IXIS PROGRAM IS NOT A RICOROUS EQUILIBRIOM NODEI. The data presented by these calculations does NOT GUARANISE that the treated gas will meet the reguired product specifications nor that any reguested level of $\mathrm{CO} 2$ slippage can be achiev'ed. please contact your ImXACO CHaMICAI representative for actual equilibrium verification. 
Sour Gas Inlet Data Sour Gas Volume System Preseure Inlet Gas Temperature Inlet Mole \& $\mathrm{CO} 2$ Inlet Mole \& H2S Inlet Gas Gravity

$\begin{aligned} \text { (MMSCFD) } & 0.075 \\ \text { (PSIA) } & 1000 \\ \text { (Deg F) } & 70 \\ \text { (MOIE \&) } & 15 \\ \text { (Mole f) } & 5 \\ \text { (AII - I) } & 0.732\end{aligned}$

Irented Gas outlet Data

$\begin{array}{lll}\text { Sweet Gas Volume (est) } & \text { (MMSCFD) } & 0.06 \\ \text { Outlet Gas Temp } & \text { (Deg E) } & 80\end{array}$

Iean Amine Data

Iype of Amine Ot1lized

MISA

Circulation Rate

Weight Percent Amine

Iean Amine Temperature

Iean Amine Ioading

(GPM)

(wt. \&)

(Deg F)

(Mol/Mol)

4.0355

18

80

0.11

Rich Amine Data

Total Acid Gas

Rich Amine Ioading

Rich Amine Temp

(Moles/hr)

(Mol/MOL)

(Deg F)

Imot Regeneration Data

stripper overhead Pressure (PSIA)

Stripper Feed Temperature (Deg F)

Reflux Temperature

Reflux Ratio

Reboiler Pressure

(Mol H2O/mol AG)

(PSIA)

Ceneral Process Temperatures

Regen Overhead Temp

Reboiler Temp

Warm Iean Amine Temp

(Deg F)

(Deg F)

(Deg F)

Entimated Exchanger Duties

Cross Exchanger Duty

Iean Cooler Duty

Condenser Duty

Reboller Duty

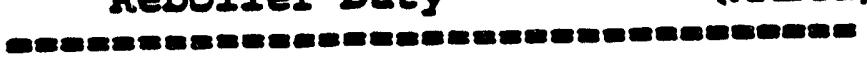

$\begin{array}{ll}\text { (MNBEu/hr) } & 0.200998 \\ \text { (MNBtu/hr) } & 0.0830351 \\ \text { (MABtu/hr) } & 0.0550283 \\ \text { (MNBEu/hr) } & 0.151184\end{array}$

1.641

0.385

104.288

This Information is not to be taken as warranty or representation for which we aseume legal responsibility. It is offered solely for your consideration, investigation and verification.

14

200

120

1.5

16

188.96

219.699

121.624

IHIS PROGRAM IS NOT A RIGOROUS EOUIIIBRIOM MODEL. The data presented by these calculations does NOT GUARANIEE that the treated gas will meet the required product specifications nor that any requested level of $\mathrm{CO} 2$ ilppage can be achieved. Please contact your TEXACO CHMMICAI representative for actual equilibrium verification. 
Quicktreat (tm) Basic Calculations

Estimated Performance

Sour Gas Inlet Data

Sour Gas Volume

System Pressure

Inlet Gas Temperature

Inlet Mole \& $\mathrm{CO} 2$

Inlet Mole \& H2S

Inlet Gas Gravity

$\begin{aligned} \text { (MMSCFD) } & 0.025 \\ \text { (PSIA) } & 1000 \\ \text { (Deg F) } & 70 \\ \text { (MOIE \&) } & 15 \\ \text { (Mole \&) } & 5 \\ \text { (AIr }=1) & 0.732\end{aligned}$

Ireated Gas Outlet Data

$\begin{array}{lll}\text { Sweet Gas Volume (est) } & \text { (MMSCFD) } & 0.02 \\ \text { outlet Gas Temp } & \text { (Deg F) } & 80\end{array}$

Ien Amise Data

Type of Arine Dtilized

Nis:

Circulation Rate

Weight Percent Amine

Iean Amine Temperature

Iean Amine Loading

$\begin{aligned} \text { (GPM) } & 1.34517 \\ \text { (Wt. t) } & 18 \\ \text { (Deg F) } & 80 \\ \text { (Mol/Mol) } & 0.11\end{aligned}$

Rich Amine Data

Total Acid Gas

Rich Amine Loading

(Moles/hr)

(Mol/MOL)

0.547

Rich Amine Temp

- (Deg F)

0.385

104.288

Input Regeneration Data

Stripper Overhead Pressure (PSIA)

14

Stripper Feed Temperature (Deg F)

200

Reflux Temperature

(Deg F)

120

Reflux Ratio

(Mol H2O/mol AG)

1.5

Reboiler Pressure

(PSIA)

16

General Process Temperatures

$\begin{array}{lll}\text { Regen Overhead Temp } & \text { (Deg F) } & 188.96 \\ \text { Reboiler Temp } & \text { (Deg F) } & 219.699 \\ \text { Warm Iean Amine Temp } & \text { (Deg F) } & 121.624\end{array}$

Estimated Exchanger Duties

$\begin{array}{lll}\text { Cross Exchasger Duty } & \text { (MBtu/hr) } & 0.0669995 \\ \text { Iean Cooler Duty } & \text { (MBtu/hr) } & 0.0276784 \\ \text { Condenser Duty } & \text { (MBtu/hr) } & 0.0183428 \\ \text { Reboiler Duty } & \text { (MBtu/hr) } & 0.0503947\end{array}$

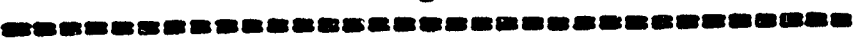

This information is not to be taken as warranty or representation for which we asoume legal responsibility. It io offered solely for your consideration, investigation and verification.

IHIS PROGRAM IS NOT A RICOROOS EOUILIBRIUM MODEI. The data prenented by these calculations does NOT GUARANISE that the treated gas will meet the required product specifications nor that any reguested level of CO2 slippage can be achieved. Please contact your TEXACO CrIMICAI representative for actual equilibrium verification. 
Quicktreat $(\mathrm{tm})$ Basic Calculations Estimated Performance

Sors Gas Inlet Data Sour Gas Volume system Pressure Inlet Gas Temperature

Inlet Mole $\mathrm{CO}_{2}$

Inlet Mole * H2S

Inlet Gas Gravity

$\begin{aligned} \text { (MMSCFD) } & 0.024 \\ \text { (PSIA) } & 1000 \\ \text { (Deg F) } & 70 \\ \text { (Mole F) } & 15 \\ \text { (Mole F) } & 5 \\ \text { (AiI } 1 \text { ) } & 0.732\end{aligned}$

Irented Gas Outlet Data Sweet Gas Volume (est) outlet Gas Temp

(MASCFD) (Deg F)

Iam Amine Data Type of Amine Otilized

Circulation Rate Weight Percent Amine Iean Amine Temperature Iean Amine Loading

Rich Amine Data Total Acid Gas Rich Amine Loading Rich Amine Temp

(Moles/hr) (Mol/Mol)

(Deg F)

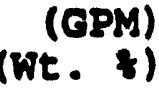
(Deg F) (MOl/MOL)

$\cos$

0.0192

$B 0$

1.29136

18

80

0.11

0.52512

0.385

104.288

Impat Regeneration Data Stripper Overhead Pressure (PSIA) 14 Stripper Feed Temperature (Deg F) 200 Ref lux Temperature Reflux Ratio Reboiler Pressure (Mol H2O/mol AG) (PSIA)

120

1.5

16

Ceneral Process Temperatures Regen Overhead Temp Reboiler Temp Warm Iean Amine Temp

(Deg F)

(Deg F)

(Deg F)

Ertimated Exchanger Duties Cross Exchanger Duty

Iean Cooler Duty

Condenser Duty Reboller Duty

\begin{tabular}{|c|c|}
\hline $\begin{array}{l}\text { (MDBtu/hr) } \\
\text { (MBBtu/hr) } \\
\text { (MBBtu/hr) } \\
\text { (MBEu/hr) }\end{array}$ & $\begin{array}{l}0.0643195 \\
0.0265712 \\
0.017609 \\
0.0483789\end{array}$ \\
\hline
\end{tabular}

188.96

219.699

121.624

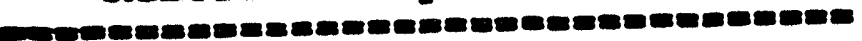

This information is not to be taken as warranty or representation for which we assume legal responsibility. It 1s offered solely for your connideration, investigation and verification.

THIS PROORAM IS NOT A RICOROOS EQUILIBRIOM MODEn. The data presented by these calculations does NOT GUARANTEE that the treated gas will meet the reguired product specifications nor that any requested level of $\mathrm{CO} 2$ elippage can be achieved. Please contact your TEXACO CHIMICAI representative for actual equilibrium verification. 
Quicktreat (tm) Basic Calculations

Estimated Performance

Sour Gas Inlet Data

Sour Gas Volume

System Pressure

Inlet Gas Temperature

Inlet Mole $\mathrm{CO} 2$

Inlet Mole \& 125

Inlet Gas Gravity

$\begin{aligned} \text { (MMSCFD) } & 0.05 \\ \text { (PSIA) } & 1000 \\ \text { (Deg F) } & 70 \\ \text { (MOIE \&) } & 15 \\ \text { (MOIE }) & 5 \\ \text { (Air - I) } & 0.732\end{aligned}$

Ireated Gas Outlet Data

Sweet Gas Volume (est)

(MASCFD)

outlet Gas Temp

(Deg F)

0.04

80

Iean Amine Data

Iype of Amine Dtilized

$\operatorname{Man}$

Circulation Rate

(GPM)

Weight Percent Amine

Iean Amine Temperature

(Wt. \&)

(Deg F)

Ieas Anine Ioading

(Mol/Mol)

2.67091

18

80

0.11

Rich Amine Data

Total Acid Gas

Rich Amine Loading

(Moles/hr)

(Mol/Mol)

Rich Amine Temp

(Deg F)

1.094

0.387

104.46

Ioput Regeneration Data

Stripper Overhead Pressure (PSIA)

Stripper Feed Temperature (Deg F)

Reflux Temperature

(Deg F)

Reflux Ratio

(Mol H20/mol AG)

(PSIA)

14

200

120

1.5

Reboiler Pressure

16

General Process Temperatures

Regen Overhead Temp (Deg F) 188.96

Reboiler Temp

Warm Iean Amine Temp

(Deg F)

229.699

(Deg F)

121.778

Estimated Exchanger Duties

Cross Exchanger Duty (MNBtu/hr) 0.132827

Iean Cooler Duty

Condenser Duty

Rebolier Duty

(MDEu/br) $\quad 0.0551622$

(MABtu/br) $\quad 0.0366855$

(MABtu/hr) $\quad 0.100592$

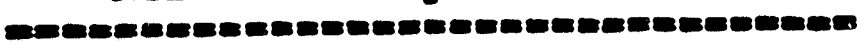

This information is not to be taken as warranty or representation for which we nsoume legal regponsibility. It is offered solely for your consideration, investigation and verification.

IHIS PROGRAY Is NOT A RICOROOS EOUIIIBRIUM MODEI. The data presented by these calculations does NOT GUARANIres that the treated gas will

meet the reguired product opecifications nor that any reguented level of CO2 elippage can be achieved. pleave contact your IExACo ctivicaI representative for actual equilibrium verification. 
Sorr Cas Inlet Data Sour Gas Volume gystem Preseure Inlet Gas Temperature Inlet Mole \& $\mathrm{CO}_{2}$ Inlet Mole $\mathrm{H2S}$ Inlet Gas Gravity

$\begin{aligned} \text { (MUSCFD) } & 1 \\ \text { (PSIA) } & 1000 \\ \text { (Deg F) } & 70 \\ \text { (MOIE }) & 15 \\ \text { (MOLE }) & 5 \\ (\text { AIX }=1) & 0.732\end{aligned}$

Ireated Gas outlet Data sweet Gas Volume (est) (Mascro) 0.8 outlet Gas Temp

(Deg F) $\quad 80.0001$

Iens Amire Data Iype of Amine Utilized

DEA

Circulation Rate Weight Percent Amine Iean Anine Temperature Irean Anine Loading

(GPM)

(wt. \&)

(Deg F) (MOI/MOI)

(Moles/br)

(Mol/Mol)

(Deg E)
28.4288

30

80

0.05

Pich Amine Data Total Acid Gas Rich Amine Loading Rich Amine Temp

$$
\text { (Deg g) }
$$

Imput Regeneration Data

Stripper Overhead Pressure (PSIA)

Stripper Feed Temperature (Deg F)

Reflux Temperature

Reflux Ratio

(Deg F)

Reboiler Presgure

(MOL H20/mol AG)
(PSIA)

21.88

0.575

115.814

\section{4 \\ 200 \\ 120 \\ 1.5 \\ 16}

General Process Temperatures

$\begin{array}{lll}\text { Regen Overhead Temp } & \text { (Deg F) } & 188.96 \\ \text { Reboiler Temp } & \text { (Deg F) } & 220.409 \\ \text { Warm Iean Amine Temp } & \text { (Deg F) } & 132.219\end{array}$

Estimated Exchanger Duties

$\begin{array}{lll}\text { Cross Exchanger Duty } & \text { (MaBtu/hr) } & 1.2475 \\ \text { Ieas Cooler Duty } & \text { (MABtu/hr) } & 0.70462 \\ \text { Condenser Duty } & \text { (Martu/hr) } & 0.73371 \\ \text { Reboiler Duty } & \text { (MaBtu/hr) } & 1.59134\end{array}$

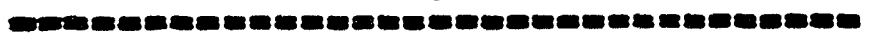

This information is not to be taken as warranty or representation for which we aseume Iegal responsibility. It is offered oolely for your consideration, investigation and verification.

IHIS PROGRAM IS NOT A RIGOROUS EOUIIIBRIUM MODEL. The data presented by these calculations does NOT GUARANTEE that the treated gas will weet the regulred product speciflcations nor that any reguected level of 002 ilippage can be achieved. Please contact your IEXACO CHIMICAI representative for actual equilibrium verification. 
Sour Gas Inlet Data Sour Gas Volume gystem Pressure Inlet Cas Temperature Inlet Mole $\mathrm{CO} 2$ Inlet Mole \& $82 \mathrm{~s}$ Inlet Gas Gravity

$\begin{aligned} \text { (MNSCFD) } & 0.75 \\ \text { (PSIA) } & 1000 \\ \text { (Deg F) } & 70 \\ \text { (Mole t) } & 15 \\ \text { (Mole t) } & 5 \\ \text { (A1工 - I) } & 0.732\end{aligned}$

Ireated Gas outlet Data

$\begin{array}{lll}\text { Sweet Gas Volume (est) (MSCFD) } & 0.6 \\ \text { Outlet Gas Teup } & \text { (Deg F) } & 80.0001\end{array}$

Iean Amine Data

Iype of Amine Utilized

Circulation Rate

Weight Percent Amine

Iean Amine Temperature

Iean Amine Loading

80.0001

Bich Amine Data

Total Acid Gas

Rich Amine Ioading

Rich Amine Temp

(Moles/hr)

(GPM)

DEA

(wt. \&)

(Deg F)

(Mol/Mol)

21.3216

30

80

0.05

(Deg F)

16.41

0.575

125.814

Imput Regeneration Data

Stripper Overhead Pressure (PSIA)

Stripper Feed Temperature (Deg F)

Reflux Temperature

(Deg F)

Reflux Ratio

(Mol H2O/mol AG)

Rebolier Pressure

(PSIA)

14

200

120

1.5

16

General Process Temperatures

Regen Overhead Teump

Reboiler Temp

Warm Ieas Amine Temp

(Deg F)

(Deg F)

188.96

220.409

(Deg F)

132.219

sotimated Exchanger Duties

Cross Exchanger Duty

Iean Cooler Duty

Condenser Duty

Reboller Duty

(MABtu/br)
(MABtu/hr)
(MBCu/br)
(MBCu/br)

0.935629

0.528465

0.550283

1.19351

ب.

This information is not to be taken as warranty or representation for which we acrume legal reoponsibility. It is offered bolely for your consideration, investigation and verification.

TEIS PROORAM Is NOT A RICOROUS EQUIIIBRION MODEI. The date presented by theoe calculations does NOT GUARANIEg that the tranted gas will meet the required product opecifications nor that any requested level of $C 02$ elippage can be achieved. please contact your Irsxaco crivicAI representative for actual equilibrium verification. 
Qulcktreat (tm) Basic Calculations Botimated Performance

Sors Gas Inlet Data Bour Gas Volume Byotem Pressure Inlet Gan Temperature

Inlet Mole \& $\mathrm{CO}_{2}$

Inlet Mole \& 123

Inlet cas Gravity

$\begin{aligned} \text { (MNSCFD) } & 0.5 \\ \text { (PSIA) } & 1000 \\ \text { (Deg F) } & 70 \\ \text { (MOIE \&) } & 15 \\ \text { (MOIE \&) } & 5 \\ \text { (Adr } 1 \text { ) } & 0.732\end{aligned}$

Ireated Gas Outlet Data

Sweet Gae Volume (est) (MuscrD) 0.4

Outlet Gas Temp

(Deg F) 80.0001

Iene Arine Data

Iype of Amine Dt1112ed

DEA

Circulation Rate

Weight Percent Amine

Iean Amine Temperature

Iean Amine Ioading

$\begin{aligned} \text { (GPM) } & 14.2144 \\ \text { (Wt. \&) } & 30 \\ \text { (Deg F) } & 80 \\ \text { (MOL/MOI) } & 0.05\end{aligned}$

Rich Amine Data

Total Acid Gas

Rich Amine Loading

Rich Amine Temp

(Moles/hr)

(Mol/Mol)

(Deg F)

10.94

0.575

115.814

Ioput Regeneration Data

Stripper Overhead Pressure (PSIA)

14

Stripper Feed Temperature (Deg F)

200

Reflux Temperature

(Deg F)

120

Reflux Ratio (Mol H2O/mol AG)

1.5

Reboller Pressure

(PSIA)

16

Eeneral Process Temperatures

Regen Overhead Temp

Reboiler Temp

Varm Ieas Amine Temp

(Deg F)

188.96

(Deg F)

220.409

(Deg F)

132.219

Evtimated Exehanger Duties

Crose Exchanger Duty

Iean Cooler Duty

(MNBtu/hr)

(MaBtu/hr)

0.623752

Condenser Duty

Reboller Duty

(MaBtu/br)

0.35231

0.366855

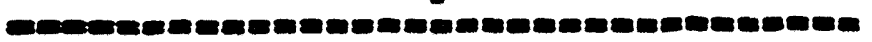

(MDEtu/br)

0.795672

This information is not to be taken as warranty or representation for which we aesume legal responsbility. It is offered solely for your consideration, investigation and verification.

THIS PROCRAM IS NOT A RICOROUS EQOILIBRIUM MODEL. The data presented by these calculations does NOT GUARANIEs that the treated gas will meet the required product opecifications nor that any requested level of $\mathrm{CO} 2$ ilppage can be achieved. Please contact your IExACO CrimICAL representative for actual equilibrium verification. 
Scour Gas Inlet Data Sour Gas Volume system Pressure Inlet Gas Temperature

Inlet Mole $\mathrm{CO} 2$

Inlet Mole \& 828

Inlet Gas Gravity

$\begin{aligned} \text { (MMSCFD) } & 0.25 \\ \text { (PSIA) } & 1000 \\ \text { (Deg F) } & 70 \\ \text { (Mole t) } & 15 \\ \text { (Mole t) } & 5 \\ \text { (AII }=1) & 0.732\end{aligned}$

Ireated Gas outlet Data

Sweet Gas Volume (eet) (MMSCFD) 0.2

Outlet Gas Temp

(Deg F) $\quad 80.0001$

Ien Aroine Data

Iype of Amise Otilized

DEA

Circulation Rate

Weight Percent Amine

Iean Anine Temperature

Iean Amine Ioading

(CPM)

(wt. \&)

(Deg F)

(MOL/MOL)

(Moles/hr)

(Mol/Mol)

(Deg F)

Rich Amine Temp

Imput Regeneration Data

stripper Overhead Pressure (PSIA)

Stripper Feed Temperature (Deg F)

Reflux Temperature

(Deg F)

Reflux Ratio

(Mol H2O/mol AG)

Reboiler Presoure

(PSIA)
7.1072

30

80

0.05

5.47

0.575

115.814

Ceneral Process Temperatures

$\begin{array}{lll}\text { Regen Overhead Temp } & \text { (Deg F) } & 188.96 \\ \text { Reboli er Temp } & \text { (Deg F) } & 220.409 \\ \text { Warm Iean Amine Temp } & \text { (Deg F) } & 132.219\end{array}$

Drimated Exchanger Duties

\begin{tabular}{|c|c|}
\hline $\begin{array}{l}\text { Coss Exchanger Duty } \\
\text { Iean Cooler Duty } \\
\text { Condenser Duty } \\
\text { Reboiler Duty }\end{array}$ & $\begin{array}{l}\text { (MABtu/br) } \\
\text { (MaBtu/hr) } \\
\text { (MBBtu/br) } \\
\text { (MABtu/br) }\end{array}$ \\
\hline
\end{tabular}

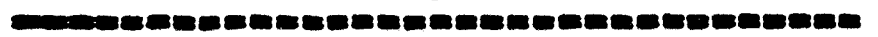

24

200

120

1.5

16

Itis information is not to be taken as warranty or representation for which we aceume legal reoponsibility. It is offered wolely for your comideration, Investigation and verification.

IXIS PROCRAY I8 NOT A RICOROUS EOUIIIBRIOM NODE. The data presented by these calculations does NOT GUARANIJE that the treated gas will meet the required product pecifications nor that any requested level

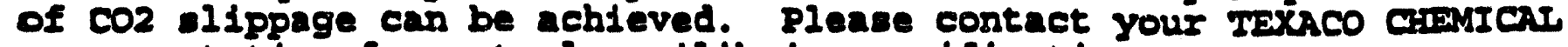
repreventative for actual equilibrium verification. 
Sour Gas Inlet Data Sour Gas Volume system Pressure Inlet Gas Temperature Inlet Mole $\mathrm{CO} 2$ Inlet Mole 425 Inlet Gas Gravity

$\begin{aligned} \text { (MDSCID) } & 0.1 \\ \text { (PSIA) } & 1000 \\ \text { (Deg F) } & 70 \\ \text { (Mole t) } & 15 \\ \text { (Mole t) } & 5 \\ \text { (AIr } 1) & 0.732\end{aligned}$

Ireated Gas Outlet Data sweet Gas Volume (est) Outlet Gas Temp

(MNSCFD)

(Deg F)

Ieno Amine Data

Iype of Anine Dtilized

Circulation Rate

Weight Percent Amine

Iean Anine Temperature

Iean Amine Ioading

Rich Amine Data

Total Acid Gas

Rich Amine Loading

Rich Amine Temp
(Moles/hr)

(Mol/Mol)

(Deg F)
0.08

80.0001

DEA

2.84288

30

80

0.05

Imput Regeneration Data

Stripper Overhead Pressure (PSIA)

Stripper Feed Temperature (Deg F)

(Deg F)

Reflux Temperature

Reflux Ratio

(Mol H2O/mol AG)

Reboiler Pressure

(PSIA)

2.188

0.575

115.814

14

200

120

1.5

26

188.96

220.409

132.219

Geoeral Process Temperatures

Regen Overhead Temp

Reboiler Temp

(Deg F)

(Deg F)

(Deg F)

Estimated Exchanger Dut1es

Cross Exchanger Duty

Iean Cooler Duty

Condenser Duty

Reboiler Duty

(MNBEu/hr)

0.12475

(MDBtu/hr)

0.070462

(MaBtu/br)

0.073371

(MABtu/br)

0.159134

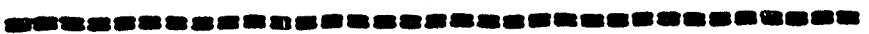

This information is not to be taken as warranty or repreventation for which we assume legal responsibility. It is offered solely for your consideration, investigation and verification.

IHIS PROGRAM IS NOT A RICOROOS DOUILIBRIOM MODEI. The data prenented by these calculations does NOT GUARANITE that the treated gas will meet the required product specifications nor that any requested level of 02 sippage can be achieved. please contact your IEXACO CrIMMICAI representative for actual equilibrium verification. 
Sour Gas Inlet Data Sour Gas Volume System Preseure Inlet Gas Temperature Inlet Mole $\mathrm{CO} 2$ Inlet Mole H2S Inlet Gas Gravity

$\begin{aligned} \text { (MMSCFD) } & 0.075 \\ \text { (PSIA) } & 1000 \\ \text { (Deg F) } & 70 \\ \text { (MOIE \&) } & 15 \\ \text { (Mole f) } & 5 \\ \text { (AIr } 1 \text { ) } & 0.732\end{aligned}$

Treated Gas Outlet Data

Sweet Gas Volume (est) (MascFD) 0.06

Outlet Gas Temp

(Deg F) $\quad 80.0001$

Iean Amine Data

Type of Amine Dtilized

DEA

Circulation Rate

Weight Percent Arine

Iean Amine Temperature

Iean Amine Loading

(QPM)

(wt. \&)

(Deg F)

(Mol/Mol)

(Moles/hr)
(Mol/Mol)
(Deg F)
2.13216

30

80

0.05

Rich Amine Data

Total Acid Gas

Rich Amine Ioading

Rich Amine Temp

Imput Regeneration Data

Stripper Overhead Pressure (PSIA)

Stripper Feed Temperature (Deg F)

(Deg 8 )

Reflux Temperature

(Mol I20/mol AG)

Reflux Ratio

(PSIA)

1.641

0.575

115.814

Reboiler Pressure

$(\operatorname{Deg} F)$
$(\operatorname{Deg} F)$
$(\operatorname{Deg} F)$

188.96

General Process Temperatures

Regen Overhead Temp

Reboiler Temp

Varm Iean Amine Temp

14

200

120

1.5

16

Estimated Exchanger Duties

Crose Exchanger Duty

Iean Cooler Duty

Condenaer Duty

Reboller Duty

(MARELA/br)

(MDBEu/hr)

(MABEu/br)

(MIBEu/br)

220.409

132.219

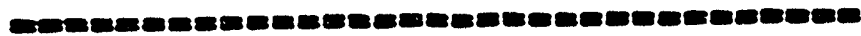

0.0935629

0.0528465

0.0550283

0.129351

This information is not to be taken as warranty or reprenentation for which we assume legal repponsibility. It is offered bolely for your consideration, investigation and verification.

IHIS PROGRAM Is IOT A RICOROOS EOUIIIBRIUM MODM. The data prepented by these calculations does NOT GUARANIys that the treated gas will meet the required product specifications nor that any reguested level of $\mathrm{CO} 2$ elippage can be achieved. plense contact your Irxaco cinilcal representative for actual equilibrium verification. 
Sour Gas Inlet Data

Sour Gas Volume

Syetem Pressure

Inlet Gas Temperature

Inlet Mole \& $\mathrm{CO} 2$

Inlet Mole $\mathrm{HzS}$

Inlet Gas Gravity

$\begin{aligned} \text { (MNSCD) } & 0.05 \\ \text { (PSIA) } & 1000 \\ \text { (Deg F) } & 70 \\ \text { (Mole \&) } & 15 \\ \text { (MOIE F) } & 5 \\ (\text { AII }=1) & 0.732\end{aligned}$

Ireated Gas Outlet Data

sweet Gas Volume (est) (MuscFD) 0.04

Outlet Gas Tẹmp

(Deg F) $\quad 80.0001$

Iean Amine Data

Iype of Amine otilized

Circulation Rate

Weight Percent Anine

Iean Amine Temperature

Iean Amine Loading

(Deg $F)$

DEA

Rich Amine Data

Total Acid Gas

Rich Amine Loading

Rich Amine Temp

$\begin{aligned} \text { (GPM) } & 1.42144 \\ \text { (Wt. F) } & 30 \\ \text { (Deg F) } & 80 \\ \text { (MOI/MOI) } & 0.05 \\ & \\ \text { (Moles/hr) } & 1.094 \\ \text { (Mol/MoI) } & 0.575 \\ \text { (Deg F) } & 115.814\end{aligned}$

Imput Regeneration Data

Stripper Overhead Pressure (PSIA)

14

Stripper Feed Temperature (Deg F) 200

Reflux Temperature

(Deg F) 120

Reflux Ratio

(MOI H2O/mol AG)

Reboiler Pressure

(PSIA) $\quad 16$

General Process Temperatures

Regen Overhead Temp

Reboiler Temp

Warm Iean Amine Temp

(Deg F)

188.96

(Deg F) 220.409

(Deg F) 132.219

Entimated Exchanger Duties

Crose Exchanger Duty

Iean Cooler buty

Condenser Duty

Reboilex Duty

(MDBtu/hr)

(MDBtu/br)

(MDBtu/br)

(NDBEu/hr)

0.0623752

0.035231

0.0366855

0.0795672

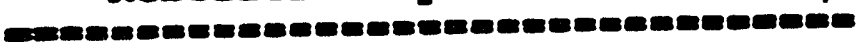

Itaie information is not to be taken as warsanty or representation for which we aseume legal remponsibility. It is offered bolely for your consideration, investigation and verification.

THIS PROGRAY Is NOT A RICOROOS IOUIIIBRIDM MODEL. The data presented by these calculations does NOT GUARANIEs that the treated gas will meet the required product pecifications nor that any requested level of 002 slippage can be achieved. please contact your IEXACO CimicAI representative for actual equilibrium verification. 
Bowr Gas Inlet Data Sour Gas Volume System Pressure Inlet Gas Temperature

Inlet Mole + $\mathrm{CO}_{2}$

Inlet Mole 1425

Inlet Gas Gravity

$\begin{aligned} \text { (MMSCFD) } & 0.025 \\ \text { (PSIA) } & 1000 \\ \text { (Deg F) } & 70 \\ \text { (MOIE \&) } & 15 \\ \text { (MOIE \&) } & 5 \\ \text { (AII }=1 \text { ) } & 0.732\end{aligned}$

Ireated Gas Outlet Data

Sweet Gas Volume (est) (MuscrD) 0.02

Outlet Gas Temp

(Deg F) B0.0001

Ienn Amine Data

Type of Amine Utilized

DEA

Circulation Rate

Weight Percent Amine

Iean Anine Temperature

Iean Amine Loading

(GPM)

(wt. \&)

(Deg F)

(MOl/MOl)

(Moles/hr)

(Mol/Mol)

(Deg F)

Rich Amine Data

Total Acid Gas

Rich Amine Loading

Rich Amine Temp

Imort Regeneration Data

Stripper Overbead Pressure (PSIA) 14

Stripper Feed Temperature (Deg F)

Reflux Temperature

Reflux Ratio

(Deg F)

Reboiler Pressure

(Mol H2O/mol AG)

(PSIA)

0.71072

30

80

0.05

0.547

0.575

125.814

Ceneral Process Temperatures

Regen Overhead Temp

Reboiler Temp

Narm Iean Anine Temp

(Deg F)

(Deg E)

(Deg E)

Eximated Exchanger Duties

Cross Exchanger Duty (MABtu/hr)

Iean Cooler Duty

Conderser Duty

Reboiler Duty
(MaBtu/hr)

(MNBtu/hr)

(MNBEu/hr)
200

120

1.5

16

188.96

220.409

132.219

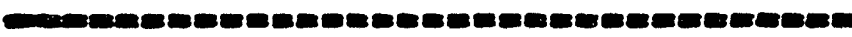

0.0311876

0.0176155

0.0283428

0.0397836

This information is not to be taken as wrranty or representation for which we aseume legal responsibility. It is offered bolely for your consideration, investigation and verification.

IHIS PROGRAM IS NOT A RICOROUS EOUILIBRIUM MODEI. The data presented by these calculations does NOT GUARANIIEE that the treated gas will meet the reguired product specifications nor that any regueated level of $\mathrm{CO} 2$ slippage can be achieved. please contact your Iraxaco crimICAI representative for actual equilibrium verification. 
Quicktreat (tm) Basic Calculations

Estimated Performance

Sour Gas Inlet Data

Sour Gas Volume

System Preseure

Inlet Gas Temperature

Inlet Mole \& $\mathrm{CO} 2$

Inlet Mole \& 325

Inlet Gas Gravity

(MUSCFD)

(PSIA)

(Deg F)

(Mole \&)

(Mole \&)

(Air - 1)

(MMSCFD)

(Deg F)

sweet Gas Volume
outlet Gas Temp

Iean Amine Data

Iype of Amine otilized

Circulation Rate

Weight Percent Amine

Lean Amine Temperature

Lean Amine Loading

Rich Amine Data

Total Acid Gas

Rich Amine Ioading

Rich Amine Temp

Ioput Regeneration Data

Stripper overhead Pressure (PSIA)

Stripper Feed Temperature (Deg F)

Reflux Temperature

Reflux Ratio

Reboiler Pressure

(Mol H20/mol AG)

(PSIA)

Eeneral Process Temperatures

Regen Overhead Temp

Reboiler Temp

varm Iean Amine Temp

Estimated Exchanger Duties

Crose Exchanger Duty
Iean Cooles Duty
Condenser Duty
Reboller Duty

(MNBtu/hr)

(MaBtu/br)

(MDBtu/br)

(MDBEu/br)

(Deg F)

(Deg F)

(Deg F)

DEA

0.682291

30

80

0.05

0.52512

0.575

125.814
14

200

120

1.5

16

188.96

220.409

132.219
0.0299401

0.0169109

0.027609

0.0381923

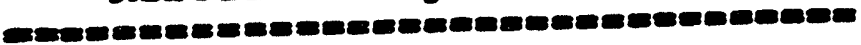

Ibis information is pot to be taken as warranty or representation for which we aveume legal responsibility. It 18 offered solely for your consideration, investigation and verification.

IHIS PROGRAM IS NOT A RICOROOS EOUIIIBRIUM MODEL. The data presented by these calculations does NOT GUARANISEs that the treated gas will meet the required product specifications nor that any requested level of Co2 slippage can be achieved. please contact your IEXACO CIMMICAI representative for actual equilibrium verification. 
11
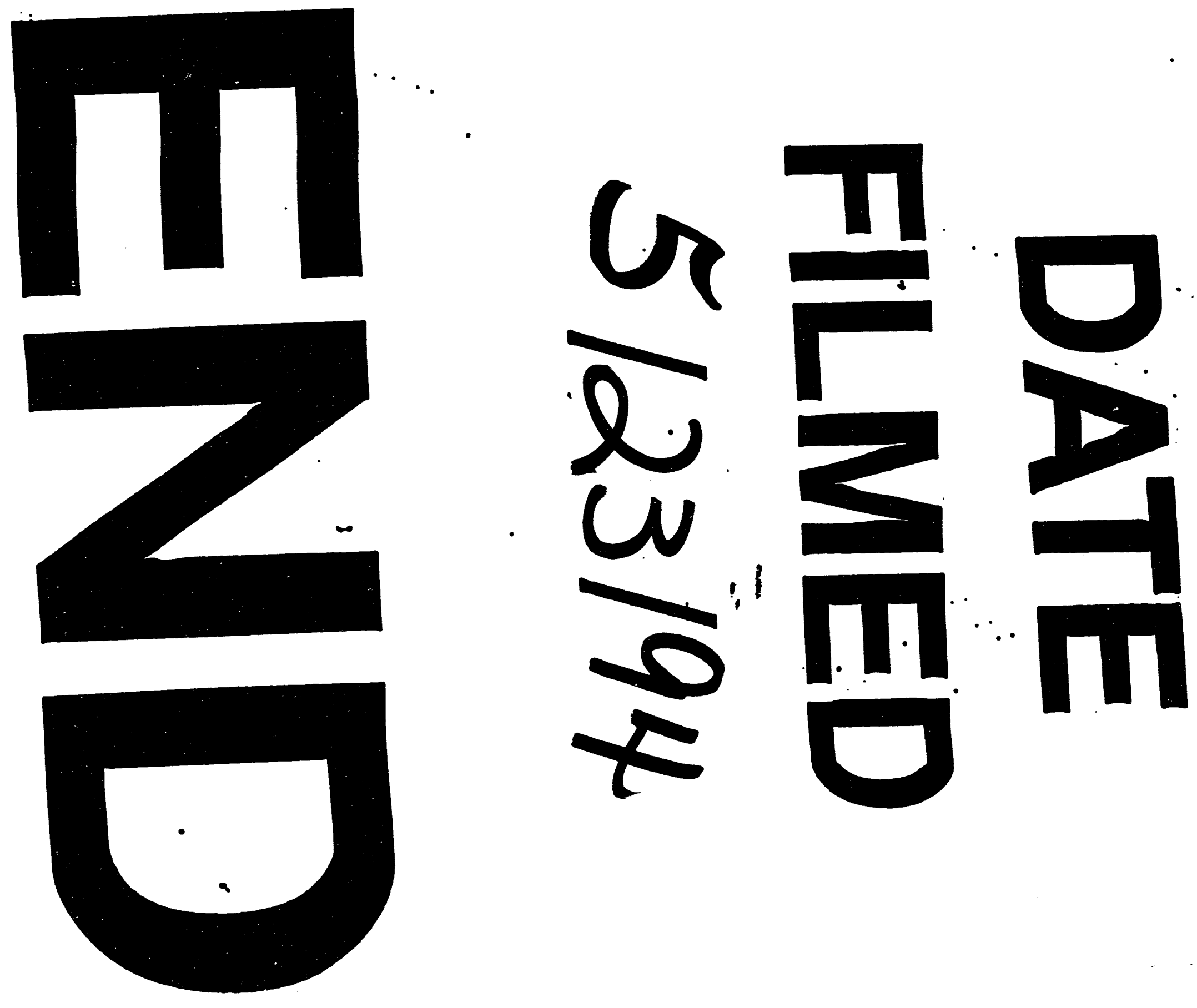


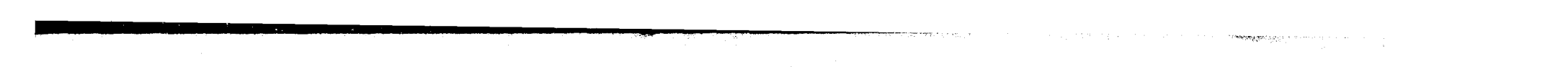

